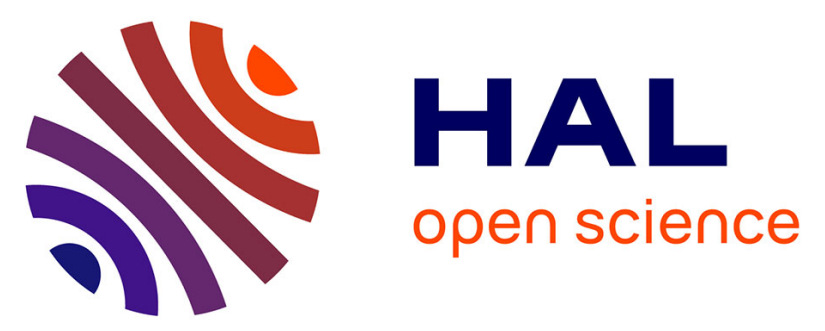

\title{
Nanostructural Characterization of Cardiolipin-Containing Tethered Lipid Bilayers Adsorbed on Gold and Silicon Substrates for Protein Incorporation
}

Marco Maccarini, Sebastian Köhler, Giovanna Fragneto, Jean-Pierre Alcaraz, Andrew Nelson, Donald Martin

\section{To cite this version:}

Marco Maccarini, Sebastian Köhler, Giovanna Fragneto, Jean-Pierre Alcaraz, Andrew Nelson, et al.. Nanostructural Characterization of Cardiolipin-Containing Tethered Lipid Bilayers Adsorbed on Gold and Silicon Substrates for Protein Incorporation. Langmuir, 2021, 37 (30), pp.8908-8923. 10.1021/acs.langmuir.1c00119 . hal-03365545

\section{HAL Id: hal-03365545 \\ https://hal.science/hal-03365545}

Submitted on 5 Oct 2021

HAL is a multi-disciplinary open access archive for the deposit and dissemination of scientific research documents, whether they are published or not. The documents may come from teaching and research institutions in France or abroad, or from public or private research centers.
L'archive ouverte pluridisciplinaire HAL, est destinée au dépôt et à la diffusion de documents scientifiques de niveau recherche, publiés ou non, émanant des établissements d'enseignement et de recherche français ou étrangers, des laboratoires publics ou privés. 


\title{
Nanostructural characterisation of cardiolipin
} containing tethered lipid bilayers adsorbed on gold and silicon substrates for protein incorporation

\author{
Sebastian Köhler, ${ }^{\dagger, \ddagger}$ Giovanna Fragneto, ${ }^{\ddagger}$ Jean-Pierre Alcaraz, ${ }^{\dagger}$ Andrew Nelson, $₫$ \\ Donald K. Martin, ${ }^{\dagger}$ and Marco Maccarini ${ }^{*} \dagger$ \\ †Université Grenoble-Alpes, Grenoble INP, TIMC-IMAG / SyNaBi (UMR 5525), 38000 \\ Grenoble, France \\ $\ddagger$ Institut Laue-Langevin, 38042 Grenoble, France \\ \ANSTO - Sydney, New Illawarra Rd, Lucas Heights NSW 2234, Australia \\ E-mail: marco.maccarini@univ-grenoble-alpes.fr
}

\begin{abstract}
A key to the development of lipid membrane based devices is a fundamental understanding of how the molecular structure of the lipid bilayer membrane is influenced by the type of lipids used to build the membrane. This is particularly important when membrane proteins are included in these devices, since the precise lipid environment affects the ability to incorporate membrane proteins and their functionality. Here we used neutron reflectometry to investigate the structure of tethered bilayer lipid membranes and to characterise the incorporation of the NhaA sodium proton exchanger in the bilayer. The lipid membranes were composed of two lipids, dioleoyl phosphatidylcholine and cardiolipin, and were adsorbed on gold and silicon substrates using two
\end{abstract}


different tethering architectures based on functionalised oligoethylene glycol molecules of different lengths. In all the investigated samples the addition of cardiolipin caused distinct structural rearrangement including crowding of ethylene glycol groups of the tethering molecules in the inner head region, and a thinning of the lipid tail region. The incorporation of NhaA in the tethered bilayers following two different protocols is quantified and the way protein incorporation modulates the structural properties of these membranes is detailed.

\section{Introduction}

Tethered bilayer lipid membranes (tBLMs) are planar lipid bilayers supported by an array of tethering molecules. ${ }^{1,2}$ These are often composed of a hydrophilic polymer whose extremities end with a group that anchors to a molecularly smooth substrate and a group that inserts into the lipid membrane. The tethering molecules serve as a framework onto which the lipid bilayer assembles. In this respect tBLMs show similarities with biological membranes characterised by the presence of cytoskeletal proteins that provide interaction and anchor

points similar to the membrane tethers. ${ }^{3}$ In addition to providing good stability, ${ }^{4-6}$ they offer a physical separation from the substrate that can accommodate extra-membraneous segments of membrane proteins incorporated in the bilayer, as well as a hydration reservoir between the bilayer and the substrate. This space enables enhanced mechanical flexibility, in contrast to solid supported lipid bilayers. ${ }^{1,7,8}$ Hence, tBLMs possess some of the fundamental properties of biological membranes, whilst also offering a simplified platform to develop lipid based bio-inspired devices.

Several types of tBLM can be found in literature that use various tethering architectures and ligands to bind to different substrates. Cornell et al. were one of the first groups to incorporate a tBLM in a functional device. ${ }^{1}$ The building blocks of that tBLM were halfmembrane-spanning phytanyl lipids tethered on gold substrates coadsorbed with polar spacer molecules composed of benzyl disulfide tetraethylene glycol to control the areal density of 
the tethered lipids. ${ }^{9,10}$ Variants of that tBLM with a double phytanoyl chain bound to the spacer ${ }^{11,12}$ or with different spacer molecules ${ }^{13}$ were also developed to enhance the flexibility of the properties of the final tBLM.

Other tBLM architectures include synthetic 1-thiahexa(ethylene oxide) lipids in combination with $\beta$-mercaptoethanol ${ }^{14}$ or 1,2-dipalmityl-3- [ $\omega$-mercaptonona(ethylene oxide)] glycerol. ${ }^{15}$ The possibility to build a tBLM from affordable and abundant components such as commercial surfactants have been explored. ${ }^{16}$ This could avoid the synthesis of complex organic molecules often needed to built tBLM and hence simplify the large scale development of tBLM-based commercial devices. tBLMs mimicking microbial outer membranes were obtained by using lipopolysaccharides extracted from Escherichia coli strains ${ }^{17}$ with an increased structural stability. tBLM with terminal deuterated anchors were also developed to exploit techniques sensitive to isotopic variation. ${ }^{18}$

More recently, novel peptide tethered lipid bilayers (pep-tBLMs) ${ }^{19,20}$ were developed in which the tethering molecules are made up by peptides. The advantage of using peptides as tethering molecules is that their length, secondary structure and hydrophobicity can be modulated by an appropriate choice of the amino acid sequence. All the previous examples concern tBLMs adsorbed on gold substrates, but by changing the binding molecule, it is possible to adapt the tBLM architecture to different substrates such as glass ${ }^{21}$ or silica. ${ }^{22-25}$

In view of their stability and versatility in terms of lipid composition, or substrate to which they are tethered, tBLMs are ideal systems to incorporate peptides, ion channels, or other membrane proteins that provide functionality to the membrane. In a previous study we already reported functional incorporation of $\mathrm{OprF}$, the main outer membrane porin of the bacterium Pseudomonas aeuruginosa into tBLMs. ${ }^{26}$ Examples of incorporation of proteins of various complexity into different kinds of tBLM can be found in the literature. Ion channels such as Gramicidin, ${ }^{1,27}$ Aquaporin ${ }^{28}$ or $\alpha$-Hemolysin ${ }^{29,30}$ have been extensively studied due to the possibility to detect their ion transport processes across the tBLM. Other studies included Neurocalcin and the Adenylate Cyclase, ${ }^{21}$ Cytochrome b5, ${ }^{25}$ Sarcolipin, ${ }^{31}$ HIV-1 Gag 
polyprotein, ${ }^{32} \mathrm{H}^{+}$Synthase, ${ }^{33}$ as well as highly complex G-protein coupled receptors. ${ }^{34,35}$ With the aid of histidine tag technology, membrane proteins contained in the bilayer can serve as the tethering molecule themselves, producing a different concept of a tBLM. This protein-tBLM system can provide further routes to increase the flexibility for reconstitution of biomimetic membranes. ${ }^{36-38}$

When trying to improve the biomimetic representation of biological membranes, and develop particular lipid membrane based devices, it is important to study the impact of different lipids on the final properties of the tBLM and how they affect the functionality of the protein inserted in the bilayer. In this work we focus on tBLMs formed from a particular combination of lipids (cardiolipin (CL) and 1,2-dioleoyl-sn-glycero-3-phosphocholine (DOPC)) into which the NhaA membrane protein, a sodium/proton exchanger found in prokaryotes, is incorporated. NhaA is a membrane integral protein with potential biotechnology implications. ${ }^{39}$ The rationale of this choice of lipids and protein follows the observation that NhaA normally exists as a dimer that it is stabilised by the presence of CL. ${ }^{40}$ This lipid, besides being implicated in the stabilisation of the protein, has interesting and peculiar features and characteristics.

CL has a unique shape with four fatty acid chains joined in two couples by a glycerol carbonyl backbone, and further connected by a glycerol backbone. This lipid is found mainly in the mitochondrial membrane of eukaryotes, or the plasma membrane of some bacteria at high fractions of $20 \% .{ }^{41}$ Its biological function is a topic of intense interest, as it seems to contribute to many processes occurring in membranes, and to the function of different membrane proteins. ${ }^{42-45}$ Deficiency of CL is related to several diseases such as Barth syndrome ${ }^{46}$ or heart failure. ${ }^{47}$ When included as a membrane component, CL influences the structural behaviour of lipid bilayers. Due its conical shape, CL has a tendency to form non-lamellar phases, ${ }^{48}$ and naturally arranges into micro domains of high negative curvature. ${ }^{49}$ When combined with other lipids, CL's effect on membrane structure is not yet completely resolved. Stabilising effects of CL on PC membranes have been reported. ${ }^{50,51}$ However, Unsay 
et al. reported a decrease in mechanical stability upon addition of CL. ${ }^{52}$ Zeczycki et al. suggested a dependence on the original packing of the membrane; when added to loosely packed membranes CL increases membrane packing, but when added to densely packed membranes the packing is decreased. ${ }^{53}$ Furthermore, an increase of bilayer thickness has been reported upon addition of tetra-myristoyl CL (TMCL) to dimyristoyl-phosphocholine (DMPC) membranes. ${ }^{54}$ The addition of tetra-oleoyl CL (TOCL) to DOPC liposome membranes reduces the overall bilayer thickness ${ }^{55}$ while increasing the thickness of the chain region.

The NhaA antiporter is an integral membrane protein that enables E. coli and other prokaryotes to adapt to high salinity and growth at alkaline $\mathrm{pH} .{ }^{56}$ The protein structure ${ }^{57}$ consists of 12 transmembrane $\alpha$-helices with the $\mathrm{N}$ - and C-terminus exposed to the cytoplasm. The cytoplasmic side shows a rough structure with loops and helices slightly protruding into the cytoplasm, whereas the periplasmic side is relatively flat. The protein has a size of 42 $\mathrm{kDa}$, consists of 388 amino-acid residues, and spans an area of approximately $40 \times 45 \AA^{2}$ in the membrane with a thickness of $50 \AA$. It transforms an $\mathrm{H}^{+}$gradient to a $\mathrm{Na}^{+}$gradient ${ }^{58}$ (or vice versa) with a stoichiometry of two protons against one sodium ion. This electrogenic ion transport is unique amongst its homologues, all of which have a symmetric stoichiometry. The ion transport properties of NhaA are strongly $\mathrm{pH}$ dependent. NhaA is inactive at $\mathrm{pH}$ lower than 6.5, but between 6.5 and 8.5 its activity rises by several orders of magnitude to a maximum turnover rate on the order of $10^{5} / \mathrm{min} .{ }^{59,60}$ These properties make NhaA very interesting for applications in nanobiotechnlology. ${ }^{61}$ A recent example showed how the incorporation of NhaA into a polyanyline supported lipid bilayer provided the basis for a high-speed rechargeable and low-energy-consuming biocapacitor for biological engineering applications. ${ }^{39}$

Previous reports have demonstrated the usefulness of neutron reflectometry (NR) in the study of the structural properties of lipid bilayer systems. NR was utilised to investigate the interaction of supported and floating bilayers with nanoengineered nanoparticles, ${ }^{62-64}$ to characterise the nanostructure of $\mathrm{tBLMs}^{65}$ and the incorporation of membrane proteins into 
lipid bilayers. ${ }^{26,66}$

The work presented in this manuscript details a series of NR experiments performed to elucidate some structural aspects of tBLMs that include the CL lipid formed by two different tethering architectures. One is the tethering architecture developed by Cornell and coworkers ${ }^{1}$ in which a benzene disulphide group provides an anchoring to a gold surface. The spacer is a short ethylene glycol segment connected to a phytanyl group that inserts in the interior of the bilayer. The lateral density of the tethering unit can be modulated by co-adsorption of short ethylene glycol segments connected to benzene disulphide groups. The other tethering architecture anchors to silicon surfaces ${ }^{67}$ through silanisation of the surface with (3-aminopropyl)triethoxysilane (APTES) molecules that produce covalent bonds with N-hydroxysuccinimide (NHS) terminated molecules, in this case a polyethylene glycol (PEG) 2000 molecule bound to a distearoyl phosphoethanolamine (DSPE) lipid. This integrates into the lipid bilayer, providing an anchor to the silicon substrate. The two architectures not only differ in the anchoring group that binds to different materials, but also in the molecular group that inserts into the lipid bilayer and the length of the ethylene glycol segment. The latter, as we will show, has a significant impact on hydration of the interstitial layer between the substrate and the bilayer and on the constraints imposed on the proximal leaflet of the bilayer, that in turn could potentially affect the ability of a protein to be incorporated and its activity.

Further, we describe several common structural changes induced by CL on both types of tBLM and how the different tBLM architectures affect the structural properties of the tethered lipid bilayer system. The study also highlights how the presence of the membrane protein NhaA, incorporated following two different protocols, modulates the structural properties of selected tBLMs. 


\section{Materials and methods}

DOPC was obtained from Avanti Polar Lipids (Alabaster, USA). APTESt and CL containing different, mostly polyunsaturated, fatty acid chains with a large fraction of linoleic acid from bovine heart and and dodecyl $\beta$-D-maltoside (DDM) were obtained from Sigma Aldrich (St. Louis, USA). Benzyl disulfide undecaethylene glycol phytanyl molecules (DLP) and benzyl disulfide tetraethylene glycol molecules (PSM) were purchased from SDx tethered membranes (Roseville, Australia). DSPE-PEG-NHS (DPN) tether molecules were purchased from Nanocs (New York, USA). Si(111) and Saphire crystal were purchased from Siltronix (Annemasse, Fr) and PI-KEM Ltd (Shropshire, UK), respectively.

\section{tBLM on gold}

Gold coated silicon or sapphire substrates were functionalised with a monolayer of DLP tether molecules and PSM. $400 \mu \mathrm{M}$ mixtures of DLP and PSM at varying ratios were deposited onto the sample surface in ethanol solution for one hour before rinsing with ethanol. Ratios of 90 \% PSM and 10\% DLP (T10) or $60 \%$ PSM and $40 \%$ DLP (T40) were used to obtain different areal densities of DLP molecules on the surface. Subsequent formation of the lipid bilayer was achieved using the rapid solvent exchange technique. ${ }^{6}$ An ethanol solution containing $5 \mathrm{mg} / \mathrm{ml}$ of the desired lipid composition was deposited onto the tether monolayer for two minutes before flushing with buffer solution, resulting in formation of the tBLM through self assembly. The whole procedure was carried out at room temperature. tBLMs produced in this way will be denoted as DLP-PSM tBLMs hereafter. The main preparation steps are depicted in Figure S1(1-4).

\section{tBLM on silicon}

Surface functionalisation for DPN tBLMs was carried out by overnight immersion of silicon substrates in 0.1 vol\% APTES in anhydrous toluene under an argon atmosphere, followed by 
ultra-sonication for 10 minutes. Subsequent curing at $120^{\circ} \mathrm{C}$ for one hour and ultra-sonication formed stable monolayers of APTES on the silicon surface. $1 \mathrm{mg} / \mathrm{ml}$ DSPE-PEG-NHS molecules dissolved in dimethylformamide were deposited onto the surface and incubated over night, followed by rinsing with ethanol, to form the monolayer of tethering molecules. Deposition of the lipid bilayer was performed using the rapid solvent exchange method. ${ }^{6}$ An ethanol solution containing $5 \mathrm{mg} / \mathrm{ml}$ of the desired lipid composition was deposited onto the functionalised surface for 10 minutes before flushing with buffer solution, resulting in formation of the tBLM through self assembly.. These tBLMs will be denoted as DPN tBLMs hereafter. The main preparation steps are depicted in Figure S1(A-F)

\section{NhaA protein production and incorporation methods}

The protein production followed a protocol based on the work of Kubicek et al. ${ }^{68}$ that we have described previously. ${ }^{39,69}$ The NhaA protein gene was introduced into the E. coli strain C43(DE3) using the plasmid vector pET15b (Novagen) for overexpression. The cells were cultivated in ampicillin containing LB medium at $37{ }^{\circ} \mathrm{C}$ until the optical density at 600 nm (OD600) reached 0.4. Next $200 \mu \mathrm{M}$ IPTG (isopropyl $\beta$-D-1-thiogalactopyranoside) was added to the culture medium to induce overexpression of the NhaA gene, followed by further cultivation for five hours. The resulting cells were harvested by centrifugation at $8000 \mathrm{rpm}$ for 5 minutes to form pellets.

For purification, the pellets were first incubated with binding buffer containing $20 \mathrm{mM}$ TRIS, $500 \mathrm{mM} \mathrm{KCl,} 10 \mathrm{mM}$ imidazole, and $12.6 \%$ (v/v) glycerol as well as lysozyme and benzonase nuclease for 30 minutes. The incubated cells were disrupted in a French pressure cell, followed by centrifugation at $14000 \mathrm{rpm}$ for 20 minutes and subsequent ultracentrifugation at $36000 \mathrm{rpm}$ for two hours to separate the membrane fraction from soluble components. The membrane fraction was then resuspended in binding buffer with additional $20 \mathrm{mM}$ DDM and incubated over night. Purification was carried out using an immobilised metal affinity chromatography column (Ni-NTA agarose, Qiagen). The column was first equilibrated with 
binding buffer followed by incubation of the membrane fraction for two hours. After rinsing with a washing buffer, the purified NhaA was recovered in an elution buffer of $20 \mathrm{mM}$ TRIS, $500 \mathrm{mM} \mathrm{KCl}, 300 \mathrm{mM}$ imidazole, $12.6 \%$ (w/v) glycerol and $225 \mu \mathrm{M}$ DDM at concentrations of 0.1 to $0.5 \mathrm{mg} / \mathrm{ml}$.

Protein incorporation into tBLMs was performed using two different methods: detergent mediated incorporation and liposome assisted incorporation. For detergent mediated incorporation the protein in elution buffer was diluted with a buffer solution containing $10 \mathrm{mM}$ HEPES and $150 \mathrm{mM} \mathrm{KCl}$ until a DDM concentration of $25 \mathrm{mM}$ was reached, which corresponds to $\approx 20 \%$ of the critical micelle concentration (CMC). ${ }^{70}$ The tBLM was incubated with this solution for 30 minutes to allow protein transfer to the tBLM, followed by thorough rinsing to remove unbound protein and detergent.

For liposome assisted incorporation, the protein in elution buffer was first transferred to liposomes. The lipids constituting the liposomes were dissolved in ethanol or chloroform, which was subsequently evaporated under a gentle nitrogen stream. This resulted in a lipid film at the bottom of the container. Buffer was added and the liquid was tip-sonicated for 3 $\times 30$ seconds or until the solution was clear. Extrusion with an Avanti Polar ${ }^{\circledR}$ mini extruder at a pore size of $400 \mathrm{~nm}$ resulted in the formation of liposomes. The protein in elution buffer was added to this at a fraction which resulted in a total DDM concentration of $20 \%$ of the CMC. To induce transfer of the protein from the detergent micelles to the liposomes, the mixture was extruded through a filter with a pore size of $100 \mathrm{~nm}$. The solution containing the liposomes with NhaA and the detergent was injected into the sample cell and incubated on the already prepared tBLM for 30 minutes to induce fusion of the proteoliposomes with the tBLM and thus transfer of the protein. After 30 minutes the sample chamber was flushed with buffer to remove any unbound components. 


\section{Neutron reflectometry}

In a NR experiment ${ }^{71}$ a neutron beam is directed toward a planar surface and the intensity of the specularly reflected beam is recorded. The ratio between the incident and reflected neutron beams is measured as a function of the component of momentum transfer perpendicular to the surface, $Q_{z}=(4 \pi / \lambda) \sin (\theta)$ where $\lambda$ is the wavelength of the incident neutron beam and $\theta$ is the angle of incidence. The reflectivity as a function of $Q_{z}$ depends on the profile along the $\mathrm{z}$ direction of the scattering length density (SLD), $\rho$, that is defined as

$$
\rho=\sum_{j} b_{j} n_{j}
$$

where $b_{j}$ and $n_{j}$ indicate the scattering length and the number per unit volume of the nuclei $j$.

One way to analyse reflectometry data involves modelling the interface as a series of parallel layers, $i$, each of which is characterised by an average scattering length density, $\rho_{i}(z)$, thickness, $T_{i}$, and roughness, $\sigma_{i}$. The parameters of this model are used to calculate

a reflectivity profile ${ }^{72-74}$ that is then compared to the measured ones in a fitting procedure where quality is assessed by using $\chi^{2}$. Uncertainties of $5 \% \Delta \chi^{2} / \chi^{2}$ are given for the single fit parameters.

A common problem in scattering experiments is the absence of a biunivocal relationship between the reflectivity profiles and the scattering length density profiles. Here we resolved this indetermination by conducting experiments on the same system with different isotopic compositions. For systems presenting aqueous sub-phases, we performed these experiments with different mixtures of $\mathrm{H}_{2} \mathrm{O}$ and $\mathrm{D}_{2} \mathrm{O}$ (contrast variation) under the assumption that the main nanostructural features of the system do not change significantly. Simultaneous co-refinement of reflectivity curves performed at different contrasts and the use of previous physical knowledge of the investigated system help to extract the most likely picture of the interface. ${ }^{75}$ Besides the scattering length densities that were fixed to the literature values 
of the corresponding substance, and fitting parameters allowed to vary within physically meaningful intervals, we enforced some constraints that reduced significantly the number of free parameters. For example, in lipid bilayers the roughness propagates between the adjacent heads and tail regions of different leaflets. In order to respect this physical feature, we made the choice to constrain the roughness to a single value for all sublayers within a lipid bilayer. Especially in the case of thinner layers such as the headgroup layers, this can sometimes bring the value of the roughness close to that of its thickness. However, this choice reduces considerably the number of free parameters in the fit based on realistic physical assumptions and accordingly it minimizes possible covariance between parameters.

The water contrasts used in this work are: $100 \% \mathrm{D}_{2} \mathrm{O}\left(\mathrm{SLD}=6.35 \cdot 10^{-6} \AA^{-2}\right), 73 \%$ $\mathrm{D}_{2} \mathrm{O}$ and $27 \% \mathrm{H}_{2} \mathrm{O}$ (gold matched water (AuMW) $\mathrm{SLD}=4.5 \cdot 10^{-6} \AA^{-2}$ ), $58 \% \mathrm{D}_{2} \mathrm{O}$ and $42 \% \mathrm{H}_{2} \mathrm{O}$ (silicon oxide matched water (OxMW) $\mathrm{SLD}=3.47 \cdot 10^{-6} \AA^{-2}$ ), $38 \% \mathrm{D}_{2} \mathrm{O}$ and $62 \% \mathrm{H}_{2} \mathrm{O}$ (silicon matched water (SiMW) $\mathrm{SLD}=2.07 \cdot 10^{-6} \AA^{-2}$ ) and $100 \% \mathrm{H}_{2} \mathrm{O}(\mathrm{SLD}=$ $\left.-0.56 \cdot 10^{-6} \AA^{-2}\right)$.

NR experiments were carried out on three neutron reflectometers: D17 and FIGARO at the Institut Laue-Langevin (Grenoble, France) and SURF at ISIS (Didcot, UK). D17 ${ }^{76,77}$ has a horizontal scattering geometry and was used in energy dispersive mode. The instrument was operated at a resolution between 1.6 and $2 \%$ with slit settings illuminating around $70 \%$ of the sample. All samples were characterised at two different angles to increase the available Q-range. Silicon substrates were measured either at $0.8^{\circ}$ and $3.0^{\circ}$, or $0.8^{\circ}$ and $3.3^{\circ}$. For sapphire substrates, $0.4^{\circ}$ and $2.8^{\circ}$ were chosen. A wavelength range from 2 to $25 \AA$ was used. This resulted in a Q-range of $0.009-0.2 \AA^{-1}$ and $0.003-0.2 \AA^{-1}$ for silicon and sapphire samples, respectively. The higher end was determined by the background of the sample. The energy dispersive reflectometer FIGARO ${ }^{78}$ has a vertical scattering geometry. It was used in the reflection up geometry, meaning that the lipid bilayers were positioned below the substrate. Experiments were performed with a $7 \% \Delta \lambda / \lambda$ resolution. Each sample was measured at two different incident angles of $0.8^{\circ}$ and $3.2^{\circ}$. With a wavelength range 
of 2-20 $\AA$, this led to a covered Q-range starting at $0.009 \AA^{-1}$ up to around $0.2 \AA^{-1}$ after which the sample background dominates the signal. The SURF reflectometer ${ }^{79}$ at the pulsed source at ISIS has a vertical scattering geometry and also operates in energy dispersive mode. Samples were characterised at three angles $0.35^{\circ}, 0.65^{\circ}$ and $1.5^{\circ}$. A wavelength range from 1-7 $\AA$ with a resolution of $4.5 \% \Delta \lambda / \lambda$ was used. The usable $Q$ space ranges from $0.012 \AA^{-1}$ to approximately $0.15 \AA^{-1}$.

In all experiments solid/liquid sample cells developed at the Institut Laue-Langevin were used. In this setup the sample surface is held against a liquid contained in a PEEK (polyether ether ketone) trough. The sample cell holds a volume of approximately $2 \mathrm{ml}$ and is connected to tubes for solvent inlet and outlet. A computer controlled HPLC pump (Smartline pump 1000) was used for automated solvent exchange. The cell temperature is controlled by a water circuit in the cell holder. Substrate blocks of surface $8 \times 5 \mathrm{~cm}^{2}$ and of $10 \mathrm{~mm}$ or 15 $\mathrm{mm}$ thickness for silicon and $15 \mathrm{~mm}$ thickness for sapphire were used. The surfaces were flat and polished to roughness smaller than $5 \AA$ by Siltronix (Annemasse, Fr). The neutron beam penetrates through the substrate block and is reflected at the inner side of the substrate on which the tBLM is formed.

NR data were analysed using Motofit ${ }^{80}$ that allows simultaneous co-refinement of reflectivity data under different isotopic contrasts. The interface is modelled by a series of parallel layers of homogeneous material characterised by an averaged SLD, a thickness, a volume fraction of aqueous medium and an interfacial roughness. Based on the interfacial layer sequence the model reflectivity profiles are calculated using the Abeles method ${ }^{72,73}$ with the roughness between two adjacent layers described by an error function. ${ }^{74}$ For some samples the lipid bilayer did not fully cover the substrate, leaving some macroscopic defects in the lipid membrane. In this case the analysis assumed that the interface is composed of two different regions having a different SLD profile (e.g. region 1: tBLM adsorbed on the substrate; region 2: tethering molecules adsorbed on the substrate). The total reflectivity is 
then a linear combination of the reflectivities of the two regions

$$
R\left(Q_{z}\right)=x_{1} R_{1}\left(Q_{z}\right)+\left(1-x_{1}\right) R_{2}\left(Q_{z}\right)
$$

where $R_{1}\left(Q_{z}\right)$ and $R_{2}\left(Q_{z}\right)$ are the reflectivities corresponding to the SLD profiles of the two

regions and $x_{1}$ is the fraction of region 1 area. This model is readily available in refnx, ${ }^{81} \mathrm{a}$ Python software that also uses the Abeles method ${ }^{72}$ to calculate the specular reflection of a stratified interface. Hereafter we will use the terms single or two area model to indicate a reflectivity profile built on one or two scattering length density profiles, respectively.

\section{Results and discussion}

Neutron reflectometry experiments were performed on two types of tBLM. DLP-PSM tBLMs adsorbed on $\mathrm{Au}$ coated silicon or sapphire substrates were characterised at two different molar ratios of DLP:PSM and different content of CL. DPN tBLMs adsorbed on silicon were studied with different content of CL. Selected samples were also studied after exposure to NhaA protein to verify and characterise the protein incorporation.

Stability up to months timescale is a well-established feature of tBLMs. ${ }^{4-6}$ In this work we verified for selected samples that the tBLMs were stable for a timeframe of several hours, which is necessary to perform the NR experiments (Supporting Information).

\section{DLP-PSM T40 tBLMs}

Figures 1 A, C and E display the reflectivity profiles at four contrasts measured for the DLPPSM T40 tBLMs at molar ratios of DOPC:CL of 90:10, 50:50 and 20:80, respectively. These data were analysed using a model built to perform the co-refinement of reflectivity data that included the substrate and the tBLM. Au-coated sapphire substrates were modelled as an infinite substrate and two slabs corresponding to layers of titanium and Au. Au coated 
silicon blocks were modelled as an infinite substrate with three slabs corresponding to a layer of native $\mathrm{SiO}_{2}$, a layer of titanium and a layer of $\mathrm{Au}$.

The tBLM was modelled with five layers representing the sub-membrane reservoir formed by the tether molecules, the inner head layer, the inner and outer tail layer and the outer head layer. A single area model was sufficient to describe the reflectivity profile, indicating that no macroscopic defects were present in the membrane. Table 1 lists the parameters obtained by fitting the model to the experimental data of Figures $1 \mathrm{~A}, \mathrm{C}, \mathrm{E}$ and the resulting SLD profiles are displayed in Figures 1 B, D, F.

The thickness of the sub-membrane layer that contains the tethering molecules is around $10 \AA$, has a water content of around $30 \%$ for all the DLP-PSM T40 tBLM samples, and is not significantly affected by the amount of CL in the lipid bilayer. As in previous studies ${ }^{26,65}$ the undecaethylene glycol in the tethering molecules are not fully extended, but instead adopt a coiled conformation. The SLD of the undecaethylene glycol was fixed at $0.6 \times 10^{-6} \AA^{-2} .^{26,65}$

The modelling of the inner head region was driven by the following consideration. This slab is composed of the headgroups of the two lipids (DOPC and CL) plus a part of the undecatethylene glycol molecule that have scattering length densities of 1.85, 2.5 and 0.6 $\times 10^{-6} \AA^{-2}$, respectively. Therefore the SLD of this region was varied during the fit between the extreme values of 0.6 and $2.5 \times 10^{-6} \AA^{-2}$. The low SLD values obtained for the inner head region suggest that it contains a significant amount of ethylene glycol molecules of the tethers. This is reasonable considering that the tethering molecules span across the inner head region, and that the sub-membrane layer is mainly composed of ethylene glycol molecules that can occupy all the interstitial space between the lipid heads. As the molar ratio of CL increases, we observe a thickening of the inner heads region. On one hand this can be explained by the heads of the CL being shifted toward the interior of the bilayer with respect to the heads of the DOPC,${ }^{54}$ hence rendering the effective thickness of the head region higher. On the other hand the trend with the molar fraction of CL suggests that the increasing portion of CL promotes some sort of crowding of the ethylene glycol molecules 
of the tether chains into the headgroups, accompanied by the expulsion of water molecules, which renders the headgroup region less hydrated than expected.

The presence of CL in the tBLM causes also a significant thinning of the lipid tail region from $\sim 30 \AA^{82}$ to $\sim 20-24 \AA$. As we will see, this is a common feature of all the tBLMs studied in this work in the presence of CL. This is the opposite behaviour observed in the case of liposomes obtained by mixing DOPC and CL, ${ }^{55}$ where the presence of CL was associated with a thicker tail region. The different conformation of the membranes (planar vs spherical aggregates) and the presence of the tethering molecules could be responsible for this different behaviour. The thickness of the outer head region is around $12 \AA$ indicating that the head groups of DOPC and CL are slightly shifted with respect to each other. ${ }^{54}$ The value of the SLD is intermediate between the value expected for CL and DOPC indicating the presence of both lipids in the outer leaflet of the lipid bilayer. The hydration of the outer head layer is also significantly higher than that of the inner head layer, as normally expected in a tethered lipid bilayer.

\section{DLP-PSM T10 tBLMs}

Figures 2 A, C display the reflectivity profiles at four contrasts measured for the DLP-PSM T10 tBLMs at molar ratios of DOPC:CL of 90:10 and 50:50, respectively. These datasets were modelled in a similar manner to the DLP-PSM T40 tBLM samples. Also in this case a single area model was sufficient to describe the reflectivity profile, indicating that no macroscopic defects were present in the membrane. Figures 2 B, D depict the scattering length density profiles and Table 2 lists the parameters obtained by the fitting analysis.

In general the DLP-PSM T10 samples show similar features to those of the DLP-PSM T40 samples described above. Despite having a larger ratio of lateral spacers our results show that there is not a simple relationship between the amount of these molecules and the properties of the sub-membrane region in terms of thickness and hydration. ${ }^{65}$ The trends observed for the characteristics of the lipid bilayer in the DLP-PSM T40 samples are also 
DOPC:CL 90:10 T40
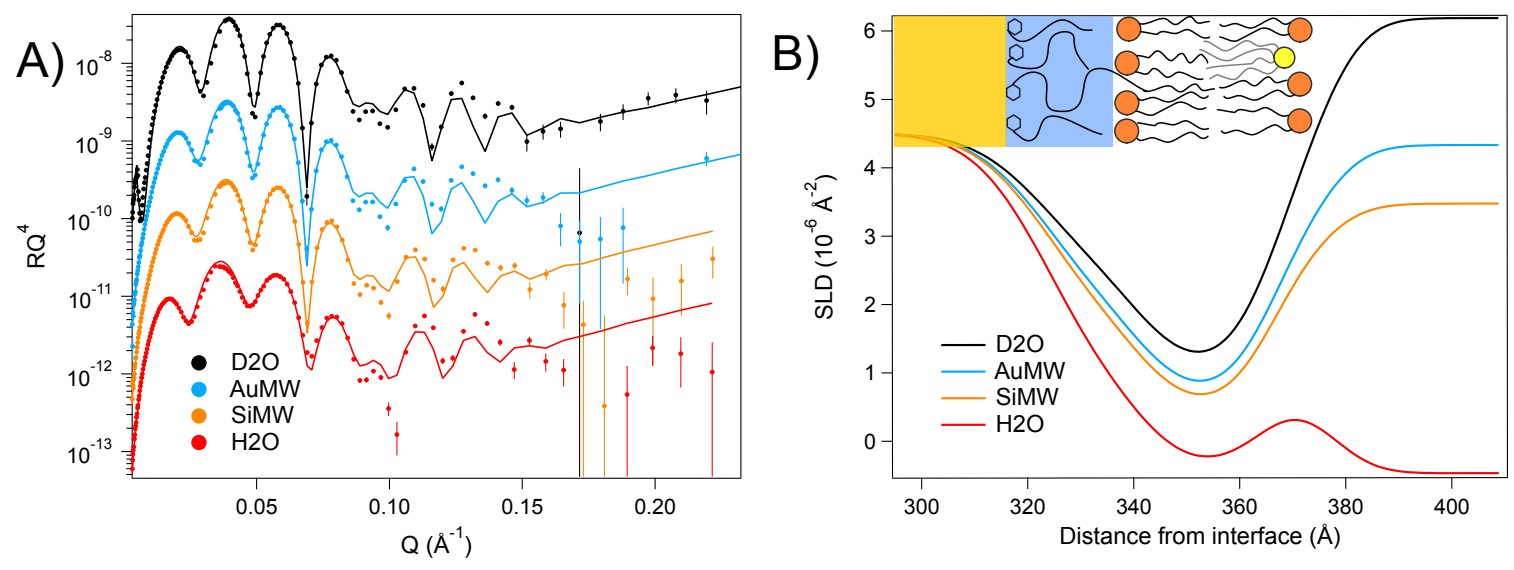

DOPC:CL 50:50 T40
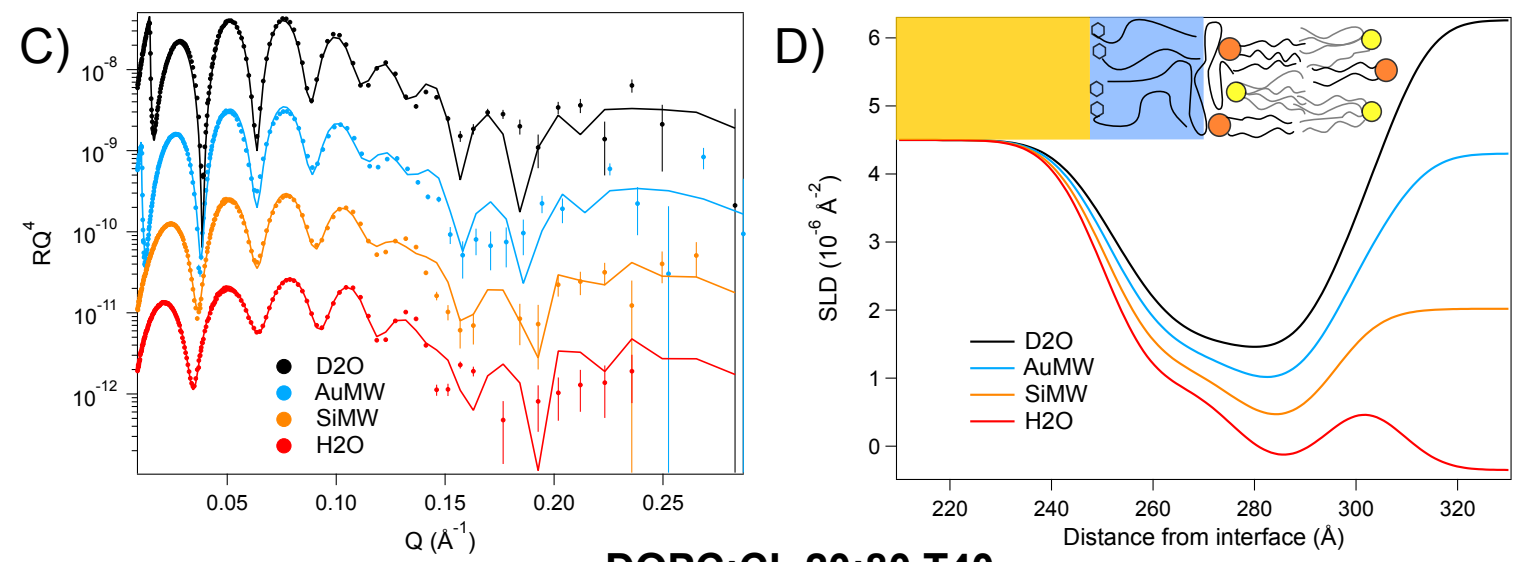

DOPC:CL 20:80 T40
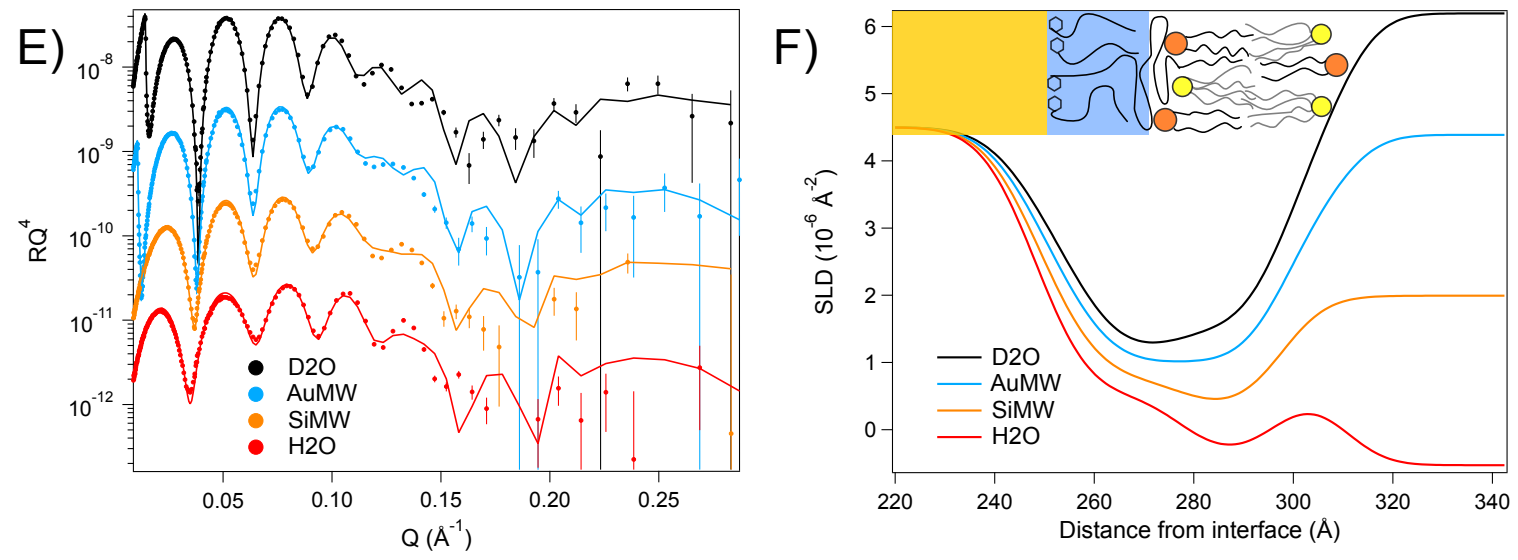

Figure 1: A) Reflectivity profiles of the DOPC:CL 90:10 T40 sample at four contrasts (symbols) and the corresponding fits (lines). B) SLD profile corresponding to the fits in (A). C) Reflectivity profiles of the DOPC:CL 50:50 T40 sample at four contrasts (symbols) and the corresponding fits (lines). D) SLD profile corresponding to the fits in (C). E) Reflectivity profiles of the DOPC:CL 20:80 T40 sample at four contrasts (symbols) and the corresponding fits (lines). F) SLD profile corresponding to the fits in (E). Data collected on D17 at the Institut Laue-Langevin. Note: the molecular cartoons do not reflect the mixing ratio of the lipids. 
Table 1: Structural parameters of the DLP-PSM T40 tBLMs corresponding to the data of Figure 1. The values without error were fixed or kept at their nominal value. Note: the SLD in the presence of the NhaA protein are listed for the case of $D_{2} O$ contrast.

\begin{tabular}{|c|c|c|c|c|}
\hline Layer & Thickness $[\AA]$ & $\mathrm{SLD}\left[\times 10^{-6} \AA^{-2}\right]$ & Solvent content [\%] & Roughness $[\AA]$ \\
\hline \multicolumn{5}{|c|}{ DOPC:CL 90:10 T40 } \\
\hline Tethers & $12 \pm 2$ & 0.6 & $27 \pm 10$ & $12 \pm 2$ \\
\hline Inner headgroup & $5 \pm 2$ & $1 \pm 1$ & $25 \pm 20$ & $7 \pm 3$ \\
\hline Inner chain region & $12 \pm 1$ & -0.3 & $22 \pm 4$ & $7 \pm 3$ \\
\hline Outer chain region & $12 \pm 1$ & -0.3 & $22 \pm 4$ & $7 \pm 3$ \\
\hline Outer headgroup & $11 \pm 3$ & $2.0 \pm 0.8$ & $45 \pm 15$ & $7 \pm 3$ \\
\hline \multicolumn{5}{|c|}{ DOPC:CL 50:50 T40 } \\
\hline Tethers & $10 \pm 1$ & 0.6 & $25 \pm 10$ & $9 \pm 2$ \\
\hline Inner headgroup & $15 \pm 1$ & $1.0 \pm 0.3$ & $10 \pm 4$ & $7 \pm 1$ \\
\hline Inner chain region & $10 \pm 1$ & -0.3 & $26 \pm 4$ & $7 \pm 1$ \\
\hline Outer chain region & $10 \pm 1$ & -0.3 & $26 \pm 4$ & $7 \pm 1$ \\
\hline Outer headgroup & $12 \pm 2$ & $1.9 \pm 0.5$ & $41 \pm 9$ & $7 \pm 1$ \\
\hline \multicolumn{5}{|c|}{ After exposure to NhaA protein } \\
\hline Tethers & $11 \pm 2$ & 0.6 & $10 \pm 9$ & $7 \pm 2$ \\
\hline Inner headgroup & $4 \pm 2$ & $1.7 \pm 1.2$ & $5 \pm 20$ & $7 \pm 1$ \\
\hline Inner chain region & $19 \pm 1$ & $1.2 \pm 0.1$ & $7 \pm 5$ & $7 \pm 1$ \\
\hline Outer chain region & $19 \pm 1$ & $1.2 \pm 0.1$ & $7 \pm 5$ & $7 \pm 1$ \\
\hline Outer headgroup & $36 \pm 5$ & $2.3 \pm 1.1$ & $68 \pm 5$ & $7 \pm 1$ \\
\hline Volume fraction protein & $0.49 \pm 0.05$ & & & \\
\hline \multicolumn{5}{|c|}{ DOPC:CL 20:80 T40 } \\
\hline Tethers & $10 \pm 1$ & 0.6 & $28 \pm 7$ & $9 \pm 2$ \\
\hline Inner headgroup & $19 \pm 1$ & $0.6 \pm 0.2$ & $10 \pm 7$ & $7 \pm 1$ \\
\hline Inner chain region & $10 \pm 1$ & -0.3 & $28 \pm 4$ & $7 \pm 1$ \\
\hline Outer chain region & $10 \pm 1$ & -0.3 & $28 \pm 4$ & $7 \pm 1$ \\
\hline Outer headgroup & $12 \pm 2$ & $1.9 \pm 0.4$ & $50 \pm 8$ & $7 \pm 1$ \\
\hline
\end{tabular}


confirmed in the case of the DLP-PSM T10 samples. The thickness of the inner head group layer increases with the amount of CL in the bilayer, and the SLD of this region decreases accordingly indicating a possible crowding of ethylene glycol molecules of the tethers in this region. The lipid tail region are compacted and the thickness and hydration of the outer head group region are higher.

The lack of a biunivocal relationship between the reflectivity curves and the scattering length density profiles could pose some level of incertitude in the determination of the structural parameters. To minimise this incertitude the experiments were performed with four contrasts, and previous physical knowledge of the system was used to set up the model that describes the scattering length density profile. To verify that the results obtained from the analysis of the reflectivity data were reasonable, we performed independent electrochemical impedance spectroscopy measurements on selected DOPC:CL 50:50 T10 samples. Starting from the structural information obtained from the NR models (namely thickness and composition of the layers corresponding to the lipid bilayers) we calculated the electrical capacitance of the membrane. This value was in reasonable agreement with capacitance values obtained from impedance spectroscopy, thus confirming the validity of our approach (see Supporting Information).

Table 2: Structural parameters of the DLP-PSM T10 tBLMs corresponding to the data of Figure 2. The values without error were fixed or kept at their nominal value.

\begin{tabular}{|c|c|c|c|c|}
\hline Layer & Thickness $[\AA]$ & $\operatorname{SLD}\left[\times 10^{-6} \AA^{-2}\right]$ & Solvent content $[\%]$ & Roughness $[\AA]$ \\
\hline \multicolumn{5}{|c|}{ "DOPC:CL 90:10 T10 } \\
\hline Tethers & $15 \pm 1$ & 0.6 & $17 \pm 3$ & $9 \pm 1$ \\
\hline Inner headgroup & $6 \pm 1$ & $0.8 \pm 0.4$ & $16 \pm 10$ & $7 \pm 1$ \\
\hline Inner chain region & $11 \pm 1$ & -0.3 & $22 \pm 2$ & $7 \pm 1$ \\
\hline Outer chain region & $11 \pm 1$ & -0.3 & $22 \pm 2$ & $7 \pm 1$ \\
\hline Outer headgroup & $9 \pm 2$ & $1.9 \pm 0.5$ & $64 \pm 7$ & $7 \pm 1$ \\
\hline \multicolumn{5}{|c|}{ DOPC:CL 50:50 T10 } \\
\hline Tethers & $11 \pm 1$ & 0.6 & $36 \pm 9$ & $7 \pm 3$ \\
\hline Inner headgroup & $18 \pm 2$ & $0.9 \pm 0.2$ & $10 \pm 7$ & $9 \pm 2$ \\
\hline Inner chain region & $10 \pm 1$ & -0.3 & $16 \pm 4$ & $9 \pm 2$ \\
\hline Outer chain region & $10 \pm 1$ & -0.3 & $16 \pm 4$ & $9 \pm 2$ \\
\hline Outer headgroup & $11 \pm 2$ & $1.9 \pm 0.6$ & $45 \pm 10$ & $9 \pm 2$ \\
\hline
\end{tabular}




\section{DOPC:CL 90:10 T10}

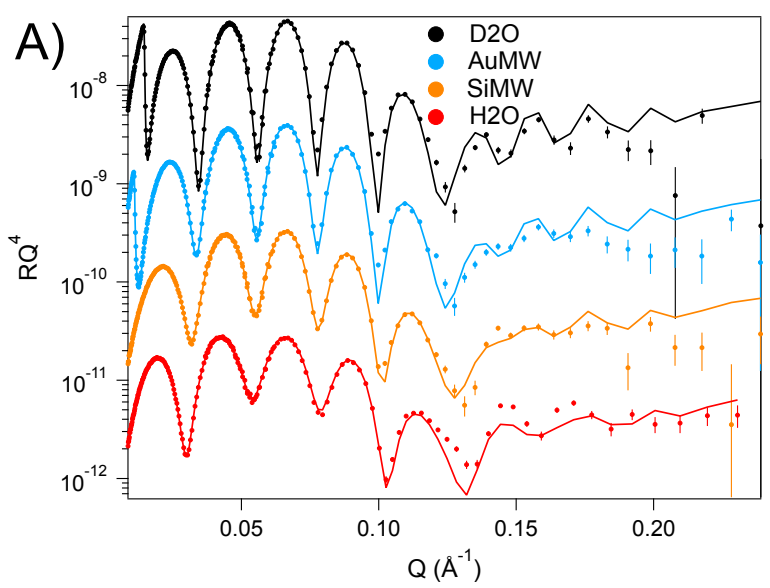

B)

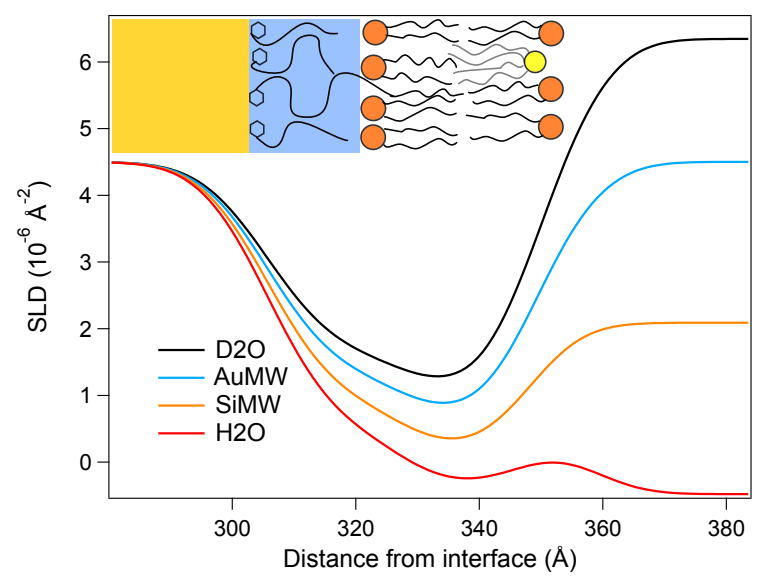

DOPC:CL 50:50 T10

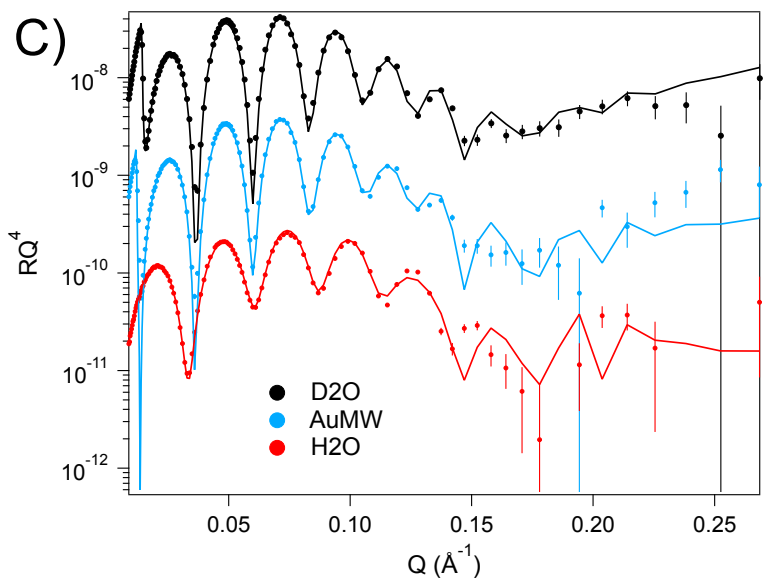

D)

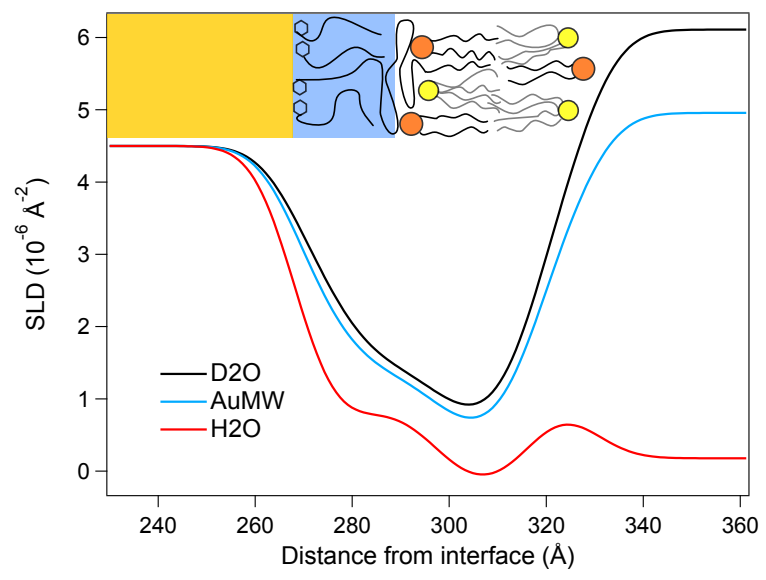

Figure 2: A) Reflectivity profiles of the DOPC:CL 90:10 T10 sample at four contrasts (symbols) and the corresponding fits (lines). B) SLD profile corresponding to the fits in (A). C) Reflectivity profiles of the DOPC:CL 50:50 T10 sample at four contrasts (symbols) and the corresponding fits (lines). D) SLD profile corresponding to the fits in (C). Data collected on D17 at the Institut Laue-Langevin. Note: the molecular cartoons do not reflect the mixing ratio of the lipids. 


\section{DPN tBLMs}

Figures 3 A, C, E display the reflectivity profiles at different contrasts measured for the DPN tBLMs at molar ratios of DOPC:CL of 100:0, 50:50 and 80:20, respectively. The silicon block that supported the tBLM was modelled as an infinite silicon block, plus a layer of native $\mathrm{SiO}_{2}$. Figures $2 \mathrm{~B}, \mathrm{D}$ and $\mathrm{F}$ depict the scattering length density profiles obtained by the fitting analysis.

For the first two samples a single area model was sufficient, suggesting that no macroscopic defects were present in the membrane. In contrast, for the 80:20 sample a single area model was not adequate for describing the reflectivity profiles (see Figure S2 in the supporting information). However, a two area model improved the fit quality significantly, indicating that macroscopic defects were present on the lipid bilayer. The numerical value of the parameters obtained by the fitting analysis are listed in Table 3. We will discuss the former two (single area model) and latter (two area model) cases separately.

The sub-membrane regions of the DPN tBLMs composed of DOPC and DOPC:CL 50:50 have a hydration level that is significantly higher than that of the DLP-PSM tBLMs adsorbed on gold, ranging from 70 to $90 \%$ with a thickness in the range of 10-20 А. Among the factors that contribute to this feature is the absence of lateral spacer molecules leaving a significant amount of space available to water molecules. Other factors that could contribute to the increase of hydration are the length of the tethering chain and the lateral density of tethering molecules. Longer tethering molecules with smaller areal density were associated with higher hydration. ${ }^{83}$ In our case the adsorption of a long ethylene glycol chain could hinder the adsorption of another tethering molecule in the vicinity of the adsorption site, effectively lowering the areal density of tethering molecules on the substrate. The thickness of the sub-membrane region, much lower that the length of the ethylene glycol chain of the tethering molecules, is also compatible with this picture, since it shows that the tethering molecules expand more in the in-plane direction rather than in the direction normal to the substrate, rendering a lower areal density of tethering molecules. 
The SLD of the inner head region is higher compared to the DLP-PSM tBLM and differs significantly from that of the ethylene glycol chains. Unlike the DLP-PSM tethers where the part of the molecule that co-localises with the inner lipid head group is composed of ethylene glycol groups (SLD $=0.6 \times 10^{-6} \AA^{-2}$ ), the DPN tethering molecules end with a DSPE lipid whose headgroup has a SLD of $2.5 \times 10^{-6} \AA^{-2}$. Considering that the SLD of the headgroup of DOPC is around $1.85 \times 10^{-6} \AA^{-2}$, the value obtained for the SLD of the inner head region $\left(1.6 \times 10^{-6} \AA^{-2}\right)$ still requires the presence of ethylene glycol groups in this region, but to a smaller extent than the DLP-PSM tBLMs. The presence of CL is associated with a thickening of the inner head region, similar to the observations in the DLP tBLMs.

The thickness of the tail region is also affected by the presence of CL. In the absence of CL it is around $30 \AA$ in agreement with literature; ${ }^{82}$ in the presence of CL the thickness reduces to lower values, consistent with the DLP-PSM tBLMs. The fact that the same thinning behaviour was observed for different tethering molecules, but only in the presence of CL, suggests that this thinning is induced by the presence of CL. The thickness and SLD of the outer head regions are similar to the values expected for DOPC or CL lipids ${ }^{55,63}$

The DPN tBLM composed of DOPC:CL 80:20 was analysed with a two area model that accounts for macroscopic areas of the substrate not covered by the lipid bilayer. The parameters describing the tethering molecule layer below the lipid bilayer (region 1) and those under the region not covered by the lipid bilayer (region 2) were not linked. The tethering molecules end with a lipid whose hydrophobic tail behaves quite differently whether it is in the presence of a lipid bilayer or exposed to an aqueous medium. In particular, the hydrophobic tail of the tether molecule would change its conformation to avoid contact with water. The first of the two structures used to represent this sample was the same tBLM model as used for the other DPN samples: a $\mathrm{SiO}_{2}$ slab at the bottom, followed by a tether slab with the SLD of PEG and four bilayer slabs representing the inner headgroup, the chain regions and the outer headgroup. The region not covered by a bilayer was modelled by a $\mathrm{SiO}_{2}$ layer that was coupled to the $\mathrm{SiO}_{2}$ layer of the first region, followed by a layer containing 
the tethering molecules whose thickness and hydration were not linked to those of the tether layer in the first region.

The reflectivity profiles and the fitting curves are displayed in Figure 3E. Figure 3F shows the SLD profiles corresponding to the part of the substrate covered with tBLM (Region 1, represented with solid lines) and the part of the substrate not covered by the lipid bilayer (Region 2, represented in dashed lines). The corresponding fitting parameters are listed in the bottom part of Table 3 .

Region 1 covers around $83 \%$ of the substrate and has a $\sim 50 \AA$ thick sub-membrane layer with a hydration hydration level of $70 \%$. Despite the properties of the sub-membrane region being very different, the parameters of the lipid bilayer itself are similar to those obtained in the other tBLMs reported in this work containing CL. They show (i) a significant increase of the thickness of the inner head region and a SLD compatible with a significant presence of ethylene glycol chains in the region; (ii) a thin hydrocarbon tail region.

Region 2 covers the remaining $17 \%$ of the sample surface that is not under the lipid bilayer and contains only the tethering molecules. The layer is thicker than the corresponding layer under the lipid membrane and it is characterised by a significantly lower hydration of 10 $\%$. We discuss a possible interpretation of the neutron reflectometry results concerning the sub-membrane region in this case. As tBLMs with similar lipid composition showed a full coverage of the substrate, this observation appears not to be related to the specific lipid composition, but rather caused by the tether molecule deposition process. DPN tBLMs require a procedure that consists of several successive steps. Prior to the grafting of the tethering molecules the silicon surface must be functionalised with the APTES silane linker. Under some circumstances the APTES molecules aggregate in a disorganised structure or multilayers instead of assembling in the form of a monolayer. ${ }^{84}$ We suspect that in this case a disorganised APTES multilayer covered the sample surface inhomogeneously and with varying roughness between the substrate and the tether layer. This caused an irregular surface distribution of the tether molecules and in turn affected the deposition of the lipid 
bilayer, resulting in an incomplete tBLM. The two regions have a significant difference in the hydration. This can be explained by considering that in order to avoid contact with water, the DSPE lipids at the end of the tethering molecules in Region 2 may hide their tails among the PEG chains, thus expelling water molecules. The SLD of the tethering region was left to vary freely to account for the presence of the DSPE lipids. The fit returned a SLD value comparable within the error to the other cases. In Region 1 on the other hand the tethering molecules can minimise the contact of the hydrophobic tails with water by integrating the DSPE into the lipid bilayer.

If we compare the structural parameters of the two different tBLM systems used in this work, the DLP-PSM and the DPN tBLMs we notice that (notwithstanding the differences in the molecule anchoring to the substrate, the length of the tethering molecules and the different segments that insert into the lipid bilayers) the characteristics of the lipid bilayers in terms of thickness, roughness and water content are very similar. The main factor that determines their structure is the ratio between the DOPC and the CL. However, there are significant differences between the two tBLM systems in the interstitial region between the proximal leaflet of the bilayer and the substrate. This region is significantly thicker and more hydrated for the DPN tBLMs. Therefore, that tBLM would be the favourable choice for inserting proteins having large regions protruding into the extramembranous region.

\section{Incorporation of NhaA protein in the tBLM}

Specific functionalities can be added to model membranes by incorporation of membrane proteins. In this work we have chosen the incorporation of the NhaA antiporter from $E$. coli whose function is to exchange one sodium ion with two protons to regulate their intra-

cellular concentration and avoid extreme alkalinisation of the cytoplasm and to contribute to the bacterium's adaptation to extreme conditions. Two different protocols of protein incorporation were utilised for the two samples, liposome assisted incorporation and detergent mediated incorporation, as explained in section Materials and Methods. 


\section{DOPC}
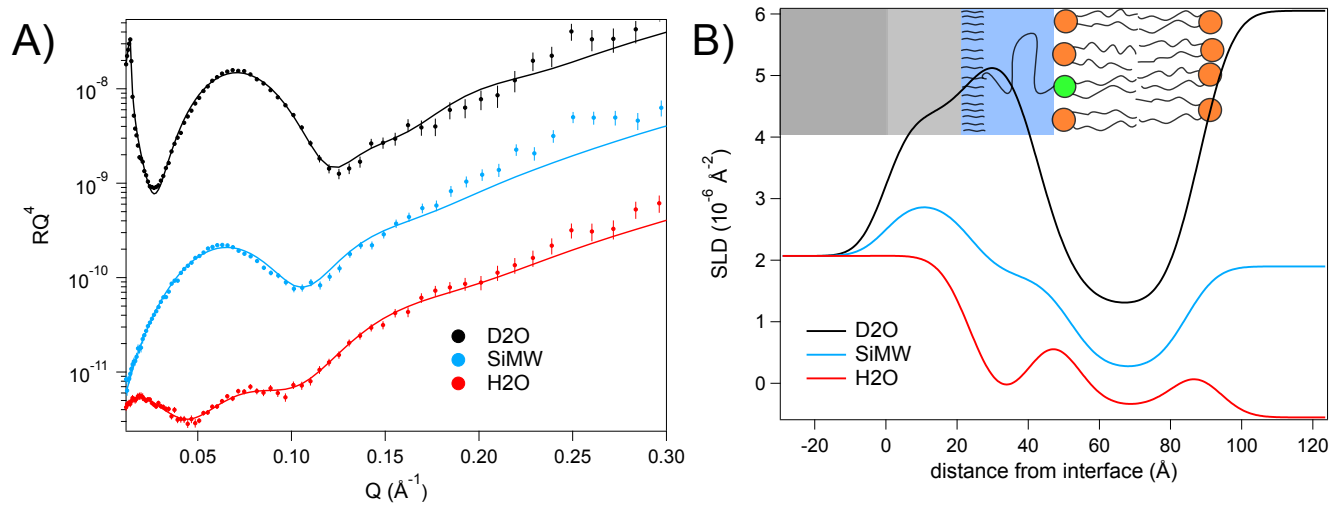

DOPC:CL 50:50
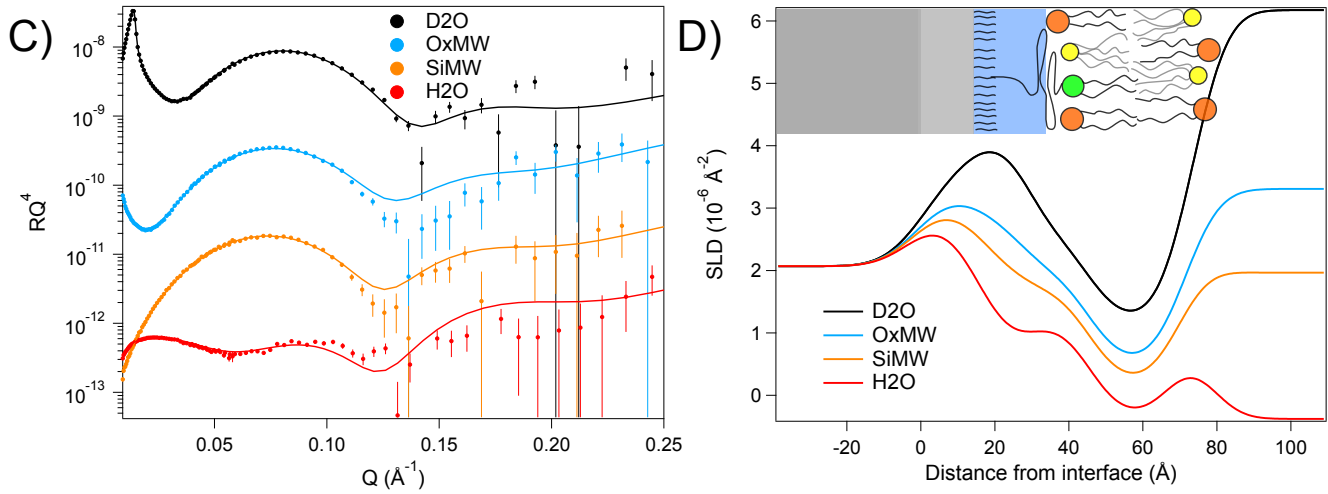

DOPC:CL 80:20
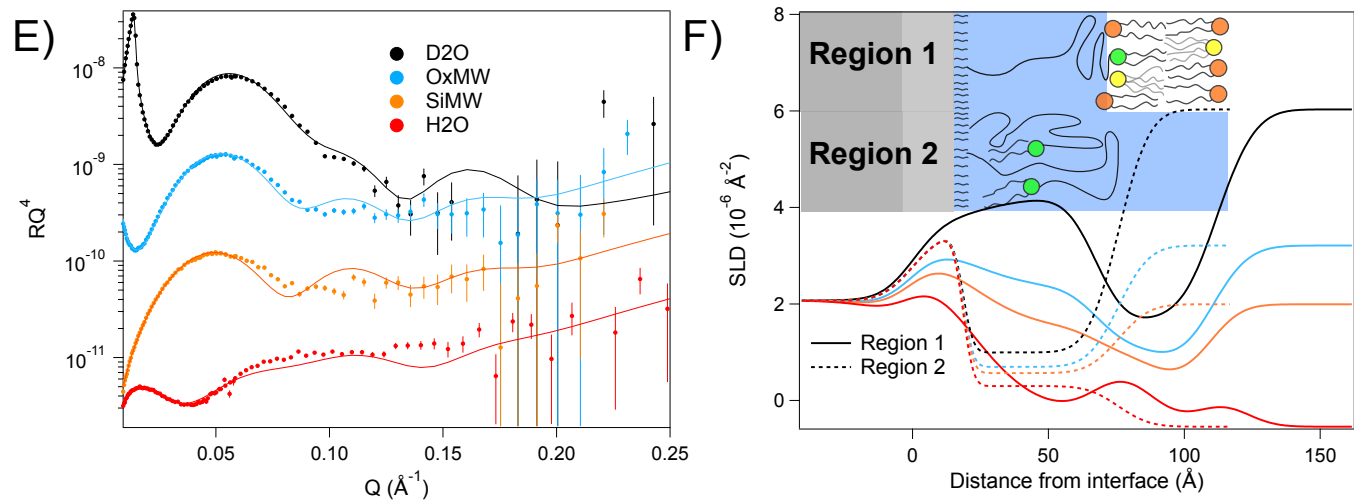

Figure 3: A) Reflectivity profiles of the DOPC DPN sample at three contrasts (symbols) and the corresponding fits (lines). B) SLD profile corresponding to the fits in (A). C) Reflectivity profiles of the DOPC:CL 50:50 DPN sample at four contrasts (symbols) and the corresponding fits (lines). D) SLD profile corresponding to the fits in (C). E) Reflectivity profiles of the DOPC:CL 80:20 DPN sample at four contrasts (symbols) and the corresponding fits (lines). F) SLD profile corresponding to the fits in (E). The two sets of lines correspond to the SLD profiles of the two regions of the mixed area model (solid lines: region 1; dashed line region 2). Data collected on SURF at the ISIS Neutron and Muon source and FIGARO at the Institut Laue-Langevin. Note: the molecular cartoons do not reflect the mixing ratio of the lipids. 
Table 3: Structural parameters of the DPN tBLMs corresponding to the data of Figure 3. The values without error were fixed or kept at their nominal value. Note that for the DOPC:CL 80:20 sample a two region model was adopted to fit the data. Note: the SLD in the presence of the NhaA protein are listed for the case of $\mathrm{D}_{2} \mathrm{O}$ contrast.

\begin{tabular}{|c|c|c|c|c|}
\hline Layer & Thickness $[\AA]$ & $\mathrm{SLD}\left[\times 10^{-6} \AA^{-2}\right]$ & Solvent content [\%] & Roughness $[\AA]$ \\
\hline \multicolumn{5}{|c|}{ DOPC } \\
\hline Tethers & $18 \pm 1$ & 0.6 & $90 \pm 3$ & $7 \pm 2$ \\
\hline Inner headgroup & $11 \pm 1$ & $1.6 \pm 0.2$ & $14 \pm 7$ & $7 \pm 1$ \\
\hline Inner chain region & $16 \pm 1$ & -0.3 & $25 \pm 2$ & $7 \pm 1$ \\
\hline Outer chain region & $16 \pm 1$ & -0.3 & $25 \pm 2$ & $7 \pm 1$ \\
\hline Outer headgroup & $6 \pm 1$ & 1.88 & $36 \pm 10$ & $7 \pm 1$ \\
\hline \multicolumn{5}{|c|}{ DOPC:CL 50:50 } \\
\hline Tethers & $12 \pm 1$ & 0.6 & $70 \pm 2$ & $11 \pm 1$ \\
\hline Inner headgroup & $17 \pm 1$ & $1.6 \pm 0.1$ & $21 \pm 3$ & $7 \pm 1$ \\
\hline Inner chain region & $12 \pm 1$ & -0.3 & $23 \pm 1$ & $7 \pm 1$ \\
\hline Outer chain region & $12 \pm 1$ & -0.3 & $23 \pm 1$ & $7 \pm 1$ \\
\hline Outer headgroup & $6 \pm 1$ & $2.4 \pm 0.2$ & $27 \pm 5$ & $7 \pm 1$ \\
\hline \multicolumn{5}{|c|}{ After exposure to NhaA protein } \\
\hline Tethers & $12 \pm 2$ & 0.6 & $85 \pm 5$ & $12 \pm 3$ \\
\hline Inner headgroup & $17 \pm 1$ & $1.8 \pm 0.3$ & $21 \pm 4$ & $7 \pm 3$ \\
\hline Inner chain region & $13 \pm 1$ & $0.3 \pm 0.1$ & $17 \pm 2$ & $7 \pm 3$ \\
\hline Outer chain region & $13 \pm 1$ & $0.3 \pm 0.1$ & $17 \pm 2$ & $7 \pm 3$ \\
\hline Outer headgroup & $7 \pm 4$ & $2.3 \pm 1.5$ & $71 \pm 14$ & $7 \pm 3$ \\
\hline Volume fraction proteins & $0.19 \pm 0.01$ & & & \\
\hline \multicolumn{5}{|c|}{ DOPC:CL 80:20 } \\
\hline \multicolumn{5}{|l|}{ Region 1} \\
\hline Tethers & $49 \pm 6$ & $0.5 \pm 0.3$ & $67 \pm 3$ & $20 \pm 1$ \\
\hline Inner headgroup & $20 \pm 7$ & $0.8 \pm 0.2$ & $14 \pm 19$ & $9 \pm 1$ \\
\hline Inner chain region & $11 \pm 3$ & -0.3 & $33 \pm 8$ & $9 \pm 1$ \\
\hline Outer chain region & $11 \pm 3$ & -0.3 & $33 \pm 8$ & $9 \pm 1$ \\
\hline Outer headgroup & $11 \pm 7$ & $1.9 \pm 1.1$ & $69 \pm 9$ & $9 \pm 1$ \\
\hline Surface coverage & $83 \% \pm 4 \%$ & & & \\
\hline \multicolumn{5}{|l|}{ Region 2} \\
\hline$\overline{\text { Tethers }}$ & $57 \pm 4$ & $0.4 \pm 0.1$ & $11 \pm 11$ & $3 \pm 5$ \\
\hline Surface coverage & $17 \% \pm 4 \%$ & & & \\
\hline
\end{tabular}


Selected samples were chosen to verify the incorporation of the NhaA protein in the tBLM. The reflectivity profiles of the DOPC:CL 50:50 DLP-PSM T40 tBLM and DOPC:CL 50:50 DPN tBLM before, and after, exposure to the NhaA protein are displayed in Figures S3 and S4 of the supporting information, respectively. Direct comparisons between the profiles before and after exposure to the protein indicate that the incorporation process causes visible changes in the reflectivity profiles of both samples. In the analysis of the reflectivity data the protein incorporation into the lipid bilayer was modelled in a similar fashion to previous work: ${ }^{26}$ NhaA was assumed to have a cylindrical shape that occupies the same volume fraction in all the different slabs of the lipid bilayer. The scattering length density of the protein was calculated by means of the Bimolecular Scattering Density Calculator ${ }^{85}$ based on the atomic composition of the protein and the estimated volume of the amino acid. The co-refinement also took into account the effect of D-H exchange of labile hydrogen atoms leading to different protein SLD in different contrasts. However, to account for the presence of possible NhaA extra-membraneous residues the thickness of the lipid head layers was allowed to vary beyond their expected limit. The reason for this choice follows the observation that adding extra layers above and below the lipid bilayer significantly complicates the model in terms of number of parameters, without improving the quality of the fit.

Table 1 lists the parameters of the DOPC:CL 50:50 DLP-PSM T40 tBLM before and after the exposure to NhaA. The liposome mediated incorporation was used for this sample. Curves corresponding to the data prior and after the incorporation are shown in Figures 1 C,D and 4 A,B, respectively. Figures 4 C,D additionally display the difference in the volume occupancy profile of the molecular components present in the tBLM before and after the protein incorporation.

Overall, the incorporation of NhaA into the DOPC:CL 50:50 DLP-PSM tBLM induced a remodelling of the lipid structure involving the thinning of the inner head group region, swelling of the lipid chain and outer headgroup regions, along with a significant reduction of the hydration in the inner region of the lipid bilayer. In more detail, we observed (i) 
DOPC:CL 90:10 T40 + NhaA
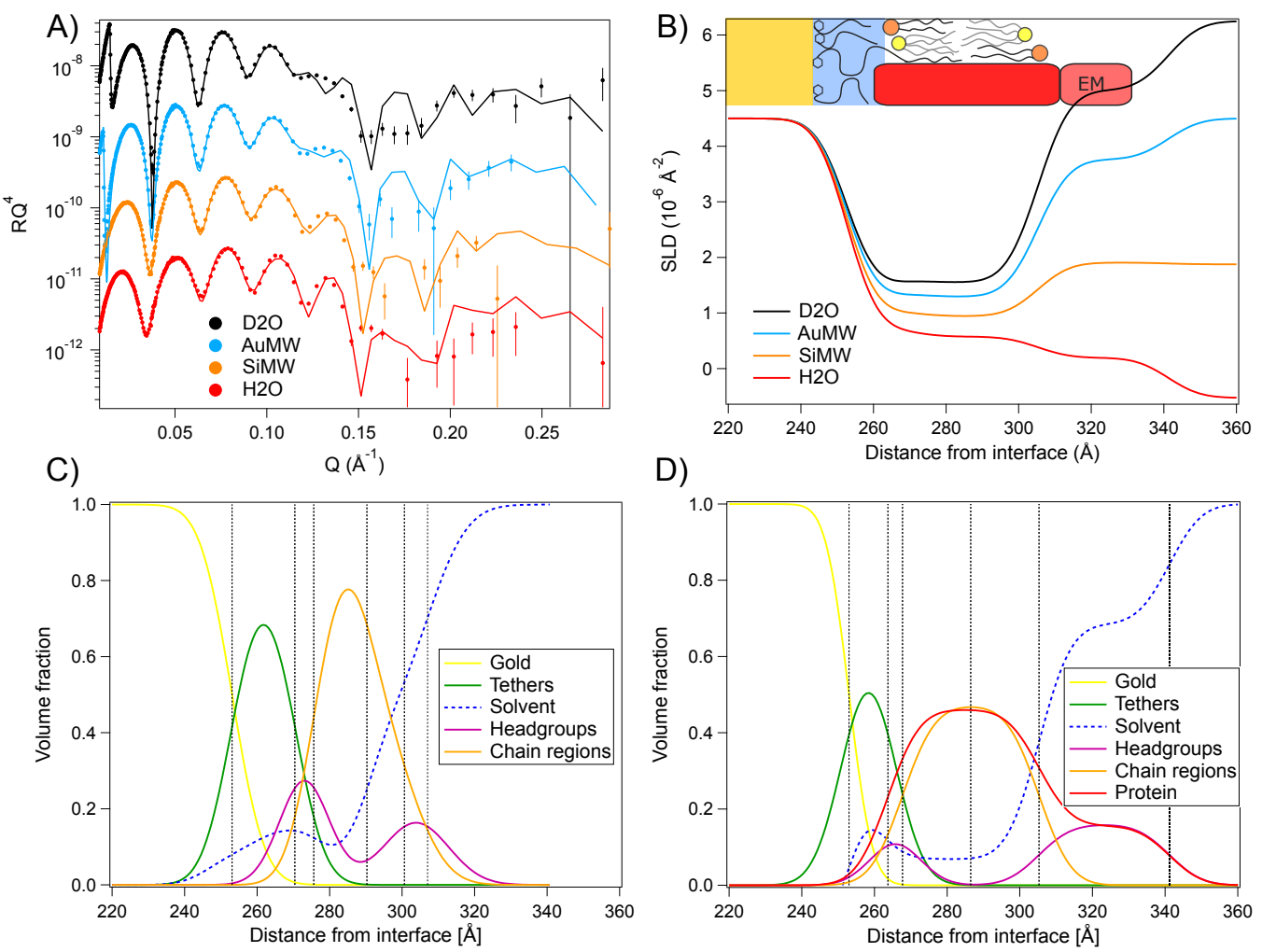

Figure 4: A) Reflectivity profiles of the DOPC:CL 50:50 DLP-PSM T40 tBLM at four contrasts (symbols) and the corresponding fits (lines) after the liposome mediated incorporation of NhaA $B$ ) $S L D$ profile corresponding to the fits in $(A)$. Volume occupancy profile of the molecular components present in the $t B L M$ before $(C)$ and after $(D)$ the liposomes mediated incorporation of NhaA. Data collected on D17 at the Institut Laue-Langevin. 
a decrease of the thickness of the inner head layer from $15 \pm 1$ to $4 \pm 2 \AA$ and an increase of its SLD from 1 to $1.7 \times 10^{-6} \AA^{-2}$; (ii) an expansion of the lipid tail regions from 10 to $19 \AA$ accompanied by a change of the SLD from -0.3 to $1.2 \times 10^{-6} \AA^{-2}$ and a reduction in the water content from 26 to $7 \%$; (iii) a thickening of the outer head region and increase of hydration.

The lipid chain regions underwent a drastic change in the SLD from a negative to a positive value. Normally the chain region has a low SLD, $-0.3 \times 10^{-6} \AA^{-2}$, because of the large number of hydrogen atoms in the hydrocarbon tail. Incorporation of a protein that has a SLD between 1.6 and $2.75 \times 10^{-6} \AA^{-2}$ (depending on the isotopic composition of the liquid phase) can significantly raise the tail region SLD, making it a very sensitive parameter to the degree of protein insertion. The SLD values of the lipid tail regions observed after the exposure to the protein indicate a protein volume fraction of $49 \pm 5 \%$ inside the bilayer. We can suppose that the incorporation of the protein in such a significant amount induced a change of conformation in the lipid tails so that the thickness of this layer became commensurate with the size of the hydrophobic part of the protein. The length of the hydrophobic zone of the NhaA protein along its axis is around $35 \AA,{ }^{86}$ a value compatible with the total thickness of the hydrophobic chain region obtained from the fits following the incorporation. The lipid chain regions experienced a significant reduction in the water content. It is reasonable to assume that in the course of the incorporation process the protein may have replaced some lipids of the tBLM, but also used the space provided by existing defects in the membrane. Either way, the lower solvent content in the chain regions after protein incorporation suggests that the incorporation may be accompanied by a repairing effect on the bilayer. This is also plausible because the protein transfer from the liposomes could include transfer of lipids from the liposome to the bilayer, that may fill pre-existing defects.

The presence of the protein in the lipid headgroup regions affected the inner and the outer leaflets differently. The decrease in thickness of the inner headgroup region could be caused 
by the presence of NhaA displacing part of the CL lipids. If the presence of CL promotes the crowding of ethylene glycol units in the headgroup region as shown in the previous section, a reduced amount of this lipid might explain the thinning of this layer. The presence of the protein might also impose a degree of order to the structure of the bilayer, further contributing to the expulsion of ethylene glycol units. The decrease in solvent content of the sub-membrane space is also consistent with the displacement of ethylene glycol units that were previously accumulated in the inner headgroup towards the sub-membrane space.

The increase of the effective thickness of the outer headgroup layer might be instead related to the histidine-tag, which is a remnant of the protein purification. This tag is assumed to protrude from the membrane, or lay on top of the bilayer surface. Even though the orientation of NhaA is not controlled in the incorporation process, the presence of such a bulky molecule on one side of the protein could induce a preferential orientation of the protein that would insert into the lipid bilayer with the histidine-group pointing in the outward direction. The opposite orientation would be less favourable due to the lack of space in the submembrane region crowded by the tethering molecules.

The data analysis was performed starting from different models and hypothesis testing criteria were followed to obtain the best fit model, as in previous work. ${ }^{26}$ Alternative models were tested such as (i) no-protein inclusion in the bilayer and (ii) protein adsorbed on the tBLM. These models failed to properly fit the reflectivity data and resulted in significantly increased values of $\chi^{2}$ (see Supporting Information)

The second sample where the protein incorporation was tested is the DOPC:CL 50:50 DPN tBLM. Table 3 lists the parameters before and after the incorporation of the NhaA protein via the detergent mediated method. The curves corresponding to the data before and after the incorporation are shown in Figures $3 \mathrm{C}, \mathrm{D}$ and $5 \mathrm{~A}, \mathrm{~B}$ respectively. Figures 5 C,D display the volume occupancy profile of the molecular components present in the tBLM before and after protein incorporation.

The most significant difference is the increase of the SLD of the inner chains from a 
negative to a positive value following the incorporation of the NhaA, similar to the DLPPSM tBLM. However, the amount of protein is significantly lower in this case, less than 20 $\%$ in volume fraction. The incorporation also induces a thickening of the hydrophobic tail region and a reduction of the water content, but to a smaller extent, from 24 to $26 \AA$ and from 23 to 17 percent, respectively. The effect of the incorporation on the other parameters is less significant than in the previous case. The magnitude of the structural effects on the tBLM seems commensurate to the amount of incorporated protein.

In this case also we tested the hypothesis of different models such as (i) no incorporation of protein or (ii) adsorption of protein on the tBLM. These alternative models were rejected since they yielded an increased value of $\chi^{2}$ (see Supporting Information).

The study described here shows a low-resolution investigation of the structural aspects of protein incorporation. We show that the reflectivity profiles are compatible with the hypothesis of proper incorporation of the NhaA protein across the lipid membrane. This is suggestive of structural integrity of the protein, since full denaturation would cause drastic changes in the structure that would be clear even at low resolution. Nonetheless, NR cannot detect minor changes in the secondary structure of the proteins that might occur after the incorporation of the protein in a lipid bilayer tethered to a solid substrate. Information on this aspect could be obtained, for example, with techniques that are sensitive to the secondary structure of a protein, such as infrared spectroscopy. Indeed, NR can be combined with attenuated total internal reflection infrared spectroscopy (ATR-IR). ${ }^{87,88}$ We can however assume structural and functional integrity of NhaA indirectly by referring to a previous work of our research group. ${ }^{39}$ NhaA produced following the same method and incorporated in a polyaniline supported lipid bilayer showed full functionality.

Our experiments showed a clear modulation of the thickness of the lipid bilayers following the NhaA incorporation. This observation is related to the so-called hydrophobic mismatch, a well-established principle that regulates the interaction between transmembrane proteins and lipid membranes, originating from the difference between the thickness of the hydrophobic 
regions of the lipid bilayer and the protein that spans across the membrane. ${ }^{89}$ This process affects significantly the organisation of lipids and proteins, but the exact implications for the structure and function strongly depend on the individual protein and the lipid system. It can induce different structural rearrangements including protein oligomerisation, protein and lipid conformational changes and membrane curvature. Generally, this process does not affect significantly the thickness of real membranes. The large variety of lipids present in real membranes allows great structural flexibility to preferentially accomodate some lipids instead of others in order to minimise the mismatch. In our case, however, the difference in the tail region thickness before and after the incorporation of the protein is significant. Among the reasons that might cause this difference is the low number of lipid species in the bilayer, that might limit the conformational ability of the bilayer to adapt to the hydrophobic domains of NhaA. Since the hydrophobic mismatch has a direct effect on the protein functionality, it is important for the development of devices based on lipid protein systems to assess the activity of the incorporated protein as a function of the lipid mixture, in view of the reduced number of lipids normally available in artificial membranes with respect to real biological ones.

The thickening of the hydrophobic regions of the lipid membrane is the result of a surface averaged quantitative determination obtained with NR through an analysis based on a slab model. This analysis however, cannot establish which molecular mechanism provides the basis of this change: for example, if it is induced by a change in the curvature of the membrane, or an extension of lipid tails or even if there is a preferential distribution of one lipid around the protein. In cases like this, more detailed molecular information could be obtained, for example, with the aid of molecular dynamics simulations that would allow to picture these structural changes at higher resolution. ${ }^{90-92}$ 
DOPC:CL 50:50 + NhaA
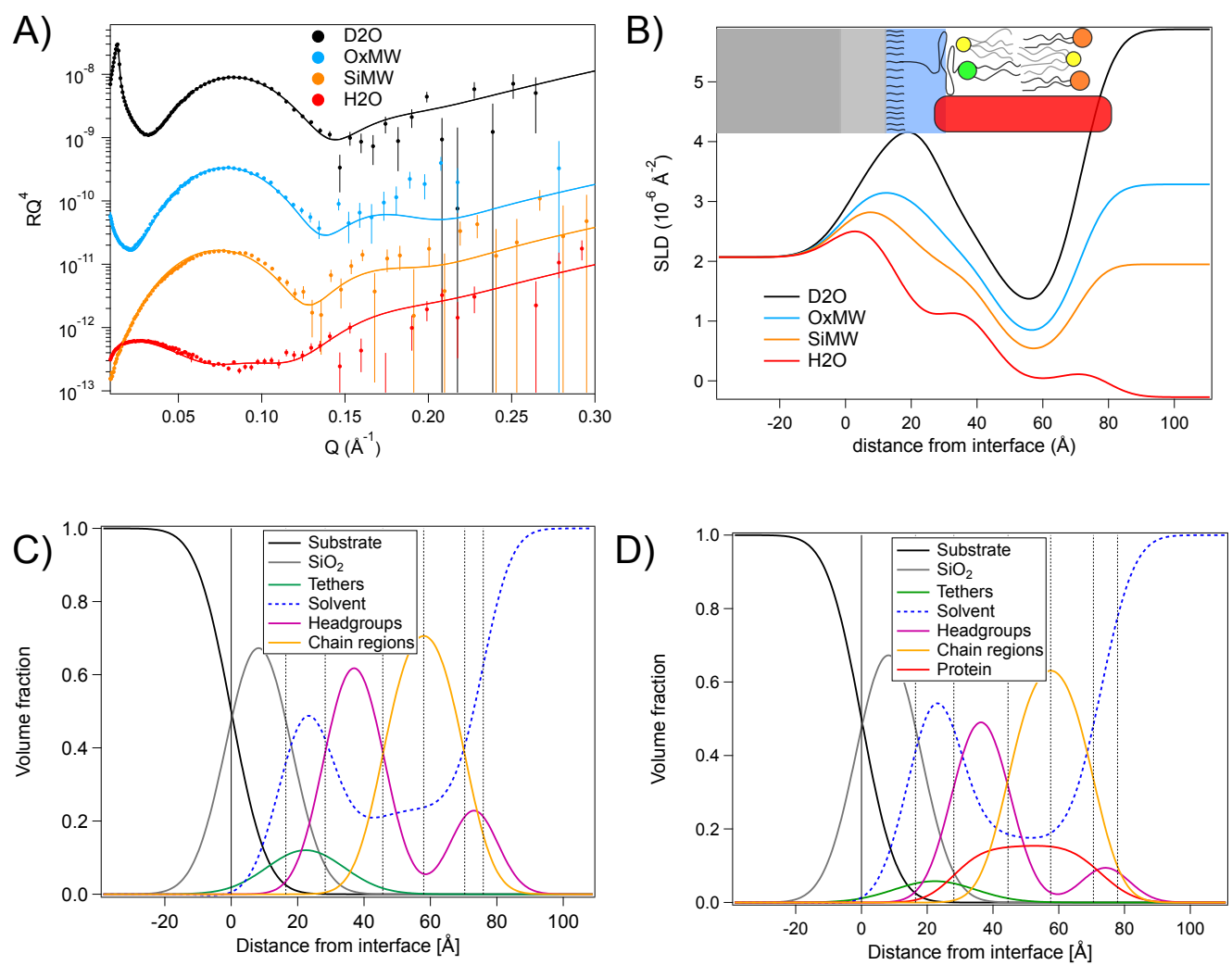

Figure 5: A) Reflectivity profiles of the DOPC:CL 50:50 DPN tBLM at four contrasts (symbols) and the corresponding fits (lines) after the detergent mediated incorporation of NhaA B) SLD profile corresponding to the fits in (A). Volume occupancy profile of the molecular components present in the $t B L M$ before $(C)$ and after $(D)$ the detergent mediated incorporation of NhaA. Data collected on FIGARO at the Institut Laue-Langevin. 


\section{Conclusions}

We provided a detailed nanostructural characterisation of three tBLM systems composed of a mixture of two lipids, DOPC and CL, adsorbed on Au and Si substrates. On Au substrates, benzyl disulfide undecaethylene glycol phytanol provided the tethering architecture whose density was modulated by co-adsorption of benzyl disulfite tetraethylene glycol molecules at two different ratios. On Si substrates, the grafting architecture was provided by adsorption of DSPC-PEG-NHS molecule on APTES monolayers. These latter tBLMs resulted in a tethering region significantly more hydrated than the former.

In all the tBLMs investigated, CL induced some distinct structural rearrangements of the membrane involving crowding of ethylene glycol groups of the tethering molecules in the inner head region, and a thinning of the lipid tail region.

Successful insertion of NhaA protein in significant amounts was demonstrated on tBLMs adsorbed on both $\mathrm{Au}$ and Si substrates. Two different methods were used to incorporate the protein; one detergent mediated, and the other liposome assisted. The analysis of neutron reflectivity experiments allowed a precise quantification of the structural changes induced by the protein to the tBLM that were commensurate to the amount of protein in the bilayer.

The results we report provide fundamental nanostructural details of a mixed phospholipid and protein bilayer system that are important for the design of functional lipid membranebased biosensors for biotechnological applications.

\section{Acknowledgement}

The work reported here was supported financially by grants from the French Agence Nationale de la Recherche (BioWATTS ANR-15-CE05-0003-01 and ImABiC ANR-16-CE190007-03) and by the PhD program of the Institut Laue-Langevin that provided allocation of neutron beam time (Experiment DOI: 10.5291/ILL-DATA.8-02-748, 10.5291/ILL-DATA.802-834) and the use of Partnership for Soft Condensed Matter laboratories. Selected mea- 
surements were performed thanks to allocation of neutron beam time provided by ISIS (UK). The authors thank Thomas Saerbeck, Samantha Micciulla, Philipp Gutfreund, Armando Maestro, and Mario Campana for performing the duties of local contacts during the experiments at ILL and ISIS. We thank Estelle Lebègue, Thomas Flinois and Valeria Italia for the technical support during selected experiments.

\section{Supporting Information Available}

The following files are available free of charge.

- SI.pdf: supporting information

\section{References}

(1) Cornell, B. A.; Braach-Maksvytis, V. L.; King, L. G.; Osman, P. D.; Raguse, B.; Wieczorek, L.; Pace, R. J. A biosensor that uses ion-channel switches. Nature 1997, $387,580-583$.

(2) Andersson, J.; Köper, I. Tethered and polymer supported bilayer lipid membranes: Structure and function. Membranes 2016, 6, 1-14.

(3) Dupuy, A. D.; Engelman, D. M. Protein area occupancy at the center of the red blood cell membrane. Proceedings of the National Academy of Sciences 2008, 105, 2848-2852.

(4) Vockenroth, I. K.; Ohm, C.; Robertson, J. W. F.; McGillivray, D. J.; Lösche, M.; Köper, I. Stable insulating tethered bilayer lipid membranes. Biointerphases 2008, 3, FA68-FA73.

(5) Prashar, J.; Sharp, P.; Scarffe, M.; Cornell, B. Making lipid membranes even tougher. Journal of Materials Research 2007, 22, 2189-2194. 
(6) Cranfield, C. G.; Cornell, B. A.; Grage, S. L.; Duckworth, P.; Carne, S.; Ulrich, A. S.; Martinac, B. Transient potential gradients and impedance measures of tethered bilayer lipid membranes: Pore-forming peptide insertion and the effect of electroporation. Biophysical Journal 2014, 106, 182-189.

(7) Plant, A.; Gueguetchkeri, M.; Yap, W. Supported phospholipid/alkanethiol biomimetic membranes: insulating properties. Biophysical Journal 2009, 67, 1126-1133.

(8) Raguse, B.; Braach-Maksvytis, V.; Cornell, B. A.; King, L. G.; Osman, P. D. J.; Pace, R. J.; Wieczorek, L. Tethered lipid bilayer membranes: formation and ionic reservoir characterization. Langmuir 1998, 14, 648-659.

(9) Krishna, G.; Schulte, J.; Cornell, B. A.; Pace, R. J.; Wieczorek, L.; Osman, P. D. Tethered Bilayer Membranes Containing Ionic Reservoirs: The Interfacial Capacitance. Langmuir 2001, 17, 4858-4866.

(10) Krishna, G.; Schulte, J.; Cornell, B. A.; Pace, R. J.; Osman, P. D. Tethered bilayer membranes containing ionic reservoirs: Selectivity and conductance. Langmuir 2003, $19,2294-2305$.

(11) Naumann, R. L.; Schiller, S. M.; Giess, F.; Grohe, B.; Hartman, K. B.; Kärcher, I.; Köper, I.; Lübben, J.; Vasilev, K.; Knoll, W. Tethered lipid bilayers on ultraflat gold surfaces. Langmuir 2003, 19, 5435-5443.

(12) Schiller, S. M.; Naumann, R.; Lovejoy, K.; Kunz, H.; Knoll, W. Archaea Analogue Thiolipids for Tethered Bilayer Lipid Membranes on Ultrasmooth Gold Surfaces. Angewandte Chemie International Edition 2003, 42, 208-211.

(13) Andersson, J.; Knobloch, J. J.; Perkins, M. V.; Holt, S. A.; Köper, I. Synthesis and Characterization of Novel Anchorlipids for Tethered Bilayer Lipid Membranes. Langmuir 2017, 33, 4444-4451. 
(14) McGillivray, D. J.; Valincius, G.; Vanderah, D. J.; Wilma, F.; Woodward, J. T.; Heinrich, F.; Kasianowicz, J. J.; Loesche, M. Molecular-scale structural and functional characterization of sparsely tethered bilayer lipid membranes. Biointerphases 2007, 2, 21-33.

(15) Heinrich, F.; Ng, T.; Vanderah, D. J.; Shekhar, P.; Mihailescu, M.; Nanda, H.; Loesche, M. A New Lipid Anchor for Sparsely Tethered Bilayer Lipid Membranes. Langmuir 2009, 25, 4219-4229.

(16) Squillace, O.; Esnault, C.; Pilard, J.-F.; Brotons, G. Electrodes for Membrane Surface Science. Bilayer Lipid Membranes Tethered by Commercial Surfactants on Electrochemical Sensors. ACS Sensors 2019, 4, 1337-1345.

(17) Andersson, J.; Fuller, M. A.; Wood, K.; Holt, S. A.; Köper, I. A tethered bilayer lipid membrane that mimics microbial membranes. Phys. Chem. Chem. Phys. 2018, 20, 12958-12969.

(18) Lee, H.-H.; Gavutis, M.; Ruzele, R., Zivileand Valiokas; Liedberg, B. Mixed SelfAssembled Monolayers with Terminal Deuterated Anchors: Characterization and Probing of Model Lipid Membrane Formation. The Journal of Physical Chemistry B 2018, 122, 8201-8210, PMID: 30085662.

(19) Naumann, R.; Baumgart, T.; Graber, P.; Jonczyk, A.; Offenhausser, A.; Knoll, W. Proton transport through a peptide-tethered bilayer lipid membrane by the $\mathrm{H}+$-ATP synthase from chloroplasts measured by impedance spectroscopy. Biosensors and Bioelectronics 2002, 17, 25-34.

(20) Chadli, M.; Rebaud, S.; Maniti, O.; Tillier, B.; Cortès, S.; Girard-Egrot, A. P. New Tethered Phospholipid Bilayers Integrating Functional G-Protein-Coupled Receptor Membrane Proteins. Langmuir 2017, 33, 10385-10401. 
(21) Rossi, C.; Homand, J.; Bauche, C.; Hamdi, H.; Ladant, D.; Chopineau, J. Differential Mechanisms for Calcium-Dependent Protein/Membrane Association as Evidenced from SPR-Binding Studies on Supported Biomimetic Membranes. Biochemistry 2003, 42, $15273-15283$.

(22) Daniel, C.; Sohn, K. E.; Mates, T. E.; Kramer, E. J.; Rädler, J. O.; Sackmann, E.; Nickel, B.; Andruzzi, L. Structural characterization of an elevated lipid bilayer obtained by stepwise functionalization of a self-assembled alkenyl silane film. Biointerphases 2007, 2, 109-118.

(23) Hertrich, S.; Stetter, F.; Rühm, A.; Hugel, T.; Nickel, B. Highly hydrated deformable polyethylene glycol-tethered lipid bilayers. Langmuir 2014, 30, 9442-9447.

(24) Atanasov, V.; Knorr, N.; Duran, R. S.; Ingebrandt, S.; Offenhäusser, A.; Knoll, W.; Köper, I. Membrane on a chip: a functional tethered lipid bilayer membrane on silicon oxide surfaces. Biophysical Journal 2005, 89, 1780-1788.

(25) Wagner, M. L.; Tamm, L. K. Tethered polymer-supported planar lipid bilayers for reconstitution of integral membrane proteins: Silane-polyethyleneglycol-lipid as a cushion and covalent linker. Biophysical Journal 2000, 79, 1400-1414.

(26) Maccarini, M.; Gayet, L.; Alcaraz, J.-P.; Liguori, L.; Stidder, B.; Watkins, E. B.; Lenormand, J.-L.; Martin, D. K. Functional Characterization of Cell-Free Expressed OprF Porin from Pseudomonas aeruginosa Stably Incorporated in Tethered Lipid Bilayers. Langmuir 2017, 9988-9996.

(27) Jadhav, S. R.; Sui, D.; Garavito, R. M.; Worden, R. M. Fabrication of highly insulating tethered bilayer lipid membrane using yeast cell membrane fractions for measuring ion channel activity. Journal of Colloid and Interface Science 2008, 322, 465-472.

(28) Coutable, A.; Thibault, C.; Chalmeau, J.; Francİois, J. M.; Vieu, C.; Noireaux, V.; Trévisiol, E. Preparation of tethered-lipid bilayers on gold surfaces for the incorporation 
of integral membrane proteins synthesized by cell-free expression. Langmuir 2014, 30, 3132-3141.

(29) McGillivray, D. J.; Valincius, G.; Heinrich, F.; Robertson, J. W. F.; Vanderah, D. J.; Febo-Ayala, W.; Ignatjev, I.; Lösche, M.; Kasianowicz, J. J. Structure of functional Staphylococcus aureus $\alpha$-hemolysin channels in tethered bilayer lipid membranes. Biophysical Journal 2009, 96, 1547-1553.

(30) Vockenroth, I. K.; Atanasova, P. P.; Jenkins, A. T. A.; Köper, I. Incorporation of $\alpha$-Hemolysin in Different Tethered Bilayer Lipid Membrane Architectures. Langmuir 2008, 24, 496-502.

(31) Becucci, L.; Guidelli, R.; Karim, C. B.; Thomas, D. D.; Veglia, G. An electrochemical investigation of sarcolipin reconstituted into a mercury-supported lipid bilayer. Biophysical Journal 2007, 93, 2678-2687.

(32) Nanda, H.; Datta, S. A.; Heinrich, F.; Loanddiesche, M.; Rein, A.; Krueger, S.; Curtis, J. E. Electrostatic interactions and binding orientation of HIV-1 matrix studied by neutron reflectivity. Biophysical Journal 2010, 99, 2516-2524.

(33) Naumann, R. L.; Baumgart, T.; Gräber, P.; Jonczyk, A.; Offenhäusser, A.; Knoll, W. Proton transport through a peptide-tethered bilayer lipid membrane by the $\mathrm{H}+$-ATP synthase from chloroplasts measured by impedance spectroscopy. Biosensors and Bioelectronics 2002, 17, 25-34.

(34) Leutenegger, M.; Lasser, T.; Sinner, E.-K.; Robelek, R. Imaging of G protein-coupled receptors in solid-supported planar lipid membranes. Biointerphases 2008, 3, FA136FA145.

(35) Robelek, R.; Lemker, E.; Wiltschi, B.; Kirste, V.; Naumann, R.; Oesterhelt, D.; Sinner, E.-K. Incorporation of In Vitro Synthesized GPCR into a Tethered Artificial Lipid Membrane System. Angewandte Chemie International Edition 2007, 46, 605-608. 
(36) Giess, F.; Friedrich, M. G.; Heberle, J.; Naumann, R. L.; Knoll, W. The ProteinTethered Lipid Bilayer: A Novel Mimic of the Biological Membrane. Biophysical Journal 2004, 87, 3213-3220.

(37) Ataka, K.; Giess, F.; Knoll, W.; Naumann, R.; Haber-Pohlmeier, S.; Richter, B.; Heberle, J. Oriented Attachment and Membrane Reconstitution of His-Tagged Cytochrome c Oxidase to a Gold Electrode. Journal of the American Chemical Society 2004, 126, 16199-16206.

(38) Hoogerheide, D. P.; Noskov, S. Y.; Kuszak, A. J.; Buchanan, S. K.; Rostovtseva, T. K.; Nanda, H. Structure of voltage-dependent anion channel-tethered bilayer lipid membranes determined using neutron reflectivity. Acta Crystallographica Section D 2018, $74,1219-1232$.

(39) Ben Tahar, A.; Zebda, A.; Alcaraz, J. P.; Gayet, L.; Boualam, A.; Cinquin, P.; Martin, D. K. A PANI supported lipid bilayer that contains NhaA transporter proteins provides a basis for a biomimetic biocapacitor. Chemical Communications 2019, 55, $13152-13155$.

(40) Gupta, K.; Donlan, J. A.; Hopper, J. T.; Uzdavinys, P.; Landreh, M.; Struwe, W. B.; Drew, D.; Baldwin, A. J.; Stansfeld, P. J.; Robinson, C. V. The role of interfacial lipids in stabilizing membrane protein oligomers. Nature 2017, 541, 421-424.

(41) Daum, G. Lipids of mitochondria. BBA - Reviews on Biomembranes 1985, 822, 1-42.

(42) Arnold, S.; Kadenbach, B. Cell respiration is controlled by ATP, an allosteric inhibitor of cytochrome-c oxidase. EUROPEAN JOURNAL OF BIOCHEMISTRY 1997, 249, $350-354$.

(43) McAuley, K. E.; Fyfe, P. K.; Ridge, J. P.; Isaacs, N. W.; Cogdell, R. J.; Jones, M. R. Structural details of an interaction between cardiolipin and an integral membrane protein. Proceedings of the National Academy of Sciences 1999, 96, 14706-14711. 
(44) Hoch, F. Minireview: Cardiolipins and mitochondrial proton-selective leakage. JOURNAL OF BIOENERGETICS AND BIOMEMBRANES 1998, 30, 511-532.

(45) Lange, C.; Nett, J.; Trumpower, B.; Hunte, C. Specific roles of protein-phospholipid interactions in the yeast cytochrome bc(1) complex structure. EMBO JOURNAL 2001, 20, 6591-6600.

(46) Schlame, M.; Ren, M. Barth syndrome, a human disorder of cardiolipin metabolism. FEBS Letters 2006, 580, 5450-5455.

(47) Saini-Chohan, H. K.; Holmes, M. G.; Chicco, A. J.; Taylor, W. A.; Moore, R. L.; McCune, S. A.; Hickson-Bick, D. L.; Hatch, G. M.; Sparagna, G. C. Cardiolipin biosynthesis and remodeling enzymes are altered during development of heart failure. Journal of Lipid Research 2009, 50, 1600-1608.

(48) Schlame, M.; Rua, D.; Greenberg, M. L. The biosynthesis and functional role of cardiolipin. Progress in Lipid Research 2000, 39, 257-288.

(49) Renner, L. D.; Weibel, D. B. Cardiolipin microdomains localize to negatively curved regions of Escherichia coli membranes. Proceedings of the National Academy of Sciences of the United States of America 2011, 108, 6264-6269.

(50) Lewis, R. N. A. H.; McElhaney, R. N. The physicochemical properties of cardiolipin bilayers and cardiolipin-containing lipid membranes. BBA - Biomembranes 2009, 1788, 2069-2079.

(51) Shibata, A.; Ikawa, K.; Shimooka, T.; Terada, H. Significant stabilization of the posphatidylcholine bilayer structure by incorporation of small amounts of cardiolipin. BBA - Biomembranes 1994, 1192, 71-78.

(52) Unsay, J. D.; Cosentino, K.; Subburaj, Y.; García-Sáez, A. J. Cardiolipin effects on membrane structure and dynamics. Langmuir 2013, 29, 15878-15887. 
(53) Zeczycki, T. N.; Whelan, J.; Hayden, W. T.; Brown, D. A.; Shaikh, S. R. Increasing levels of cardiolipin differentially influence packing of phospholipids found in the mitochondrial inner membrane. Biochemical and Biophysical Research Communications 2014, 450, 366-371.

(54) Boscia, A. L.; Treece, B. W.; Mohammadyani, D.; Klein-Seetharaman, J.; Braun, A. R.; Wassenaar, T. A.; Klösgen, B.; Tristram-Nagle, S. X-ray structure, thermodynamics, elastic properties and MD simulations of cardiolipin/dimyristoylphosphatidylcholine mixed membranes. Chemistry and Physics of Lipids 2014, 178, 1-10.

(55) Pan, J.; Cheng, X.; Sharp, M.; Ho, C.-S.; Khadka, N.; Katsaras, J. Structural and mechanical properties of cardiolipin lipid bilayers determined using neutron spin echo, small angle neutron and X-ray scattering, and molecular dynamics simulations. Soft Matter 2015, 11, 130-138.

(56) Padan, E.; Bibi, E.; Ito, M.; Krulwich, T. A. Alkaline pH homeostasis in bacteria: New insights. BBA - Biomembranes 2005, 1717, 67-88.

(57) Hunte, C.; Screpanti, E.; Venturi, M.; Rimon, A.; Padan, E.; Michel, H. Structure of a $\mathrm{Na}+/ \mathrm{H}+$ antiporter and insights into mechanism of action and regulation by $\mathrm{pH}$. Nature 2005, 435, 1197-1202.

(58) Padan, E. The enlightening encounter between structure and function in the NhaA $\mathrm{Na}+-\mathrm{H}+$ antiporter. Trends in Biochemical Sciences 2008, 33, 435-443.

(59) Mager, T.; Rimon, A.; Padan, E.; Fendler, K. Transport mechanism and pH regulation of the $\mathrm{Na}+/ \mathrm{H}+$ antiporter NhaA from Escherichia coli: An electrophysiological study. Journal of Biological Chemistry 2011, 286, 23570-23581.

(60) Taglicht, D.; Padan, E.; Schuldiner, S. Overproduction and Purification of a Functional $\mathrm{Na}+/ \mathrm{H}+$ Antiporter Coded by nhaA (ant) from Escherichia coli. The Journal of Biological Chemistry 1991, 266, 11289-11294. 
(61) Martin, D. K. Nanobiotechnology of Biomimetic Membranes; Springer, 2007; Vol. 1; pp $1-21$.

(62) Tatur, S.; Maccarini, M.; Barker, R.; Nelson, A.; Fragneto, G. Effect of Functionalized Gold Nanoparticles on Floating Lipid Bilayers. LANGMUIR 2013, 29, 6606-6614.

(63) Di Silvio, D.; Maccarini, M.; Parker, R.; Mackie, A.; Fragneto, G.; Baldelli Bombelli, F. The effect of the protein corona on the interaction between nanoparticles and lipid bilayers. Journal of Colloid and Interface Science 2017, 504, 741-750.

(64) Lolicato, F.; Joly, L.; Martinez-Seara, H.; Fragneto, G.; Scoppola, E.; Baldelli Bombelli, F.; Vattulainen, I.; Akola, J.; Maccarini, M. The Role of Temperature and Lipid Charge on Intake/Uptake of Cationic Gold Nanoparticles into Lipid Bilayers. Small 2019, 15.

(65) Maccarini, M.; Watkins, E. B.; Stidder, B.; Alcaraz, J.-P.; Cornell, B. A.; Martin, D. K. Nanostructural determination of a lipid bilayer tethered to a gold substrate. The European Physical Journal E 2016, 39, 123.

(66) Soranzo, T.; Martin, D. K.; Lenormand, J. L.; Watkins, E. B. Coupling neutron reflectivity with cell-free protein synthesis to probe membrane protein structure in supported bilayers. Scientific Reports 2017, 7, 1-10.

(67) Fragneto, G.; Delhom, R.; Joly, L.; Scoppola, E. Neutrons and Model Membranes: Moving towards Complexity. Current Opinion in Colloid \& Interface Science 2018,

(68) Kubicek, J.; Block, H.; Maertens, B.; Spriestersbach, A.; Labahn, J. Expression and purification of membrane proteins. Methods in Enzymology 2014, 541, 117-140.

(69) Flinois, T.; Lebègue, E.; Zebda, A.; Alcaraz, J. P.; Martin, D. K.; Barrière, F. Assisted lipid deposition by reductive electrochemical aryldiazonium grafting and insertion of 
the antiport NhaA protein in this stable biomimetic membrane. Colloids and Surfaces B: Biointerfaces 2020, 190, 110924.

(70) Martin, D. K.; Cornell, B. A.; Liguori, L.; Cinquin, P.; Liguori, L.; Lenormand, J.-L.; Alcaraz, J.-P.; Scolan, G. Liposomes, Lipid Bilayers and Model Membranes From Basic Research to Application; 2016; pp 383-400.

(71) Penfold, J.; Thomas, R. K. The Application of the Specular Reflection of Neutrons to the Study of Surfaces and Interfaces. Journal of Physics: Condensed Matter 1990, 2, 1369-1412.

(72) Heavens, O. Optical Properties of Thin Films. Reports on Progress in Physics 1960, 23, 1-65.

(73) Abelès, F. La théorie générale des couches minces. J. Phys. le Radium 1950, 11, 307309.

(74) Névot, L.; Croce, P. Caractérisation des surfaces par réflexion rasante de rayons X. Application à l'étude du polissage de quelques verres silicates. Rev. Phys. Appl. (Paris) 1980, 15, 761-779.

(75) Crowley, T.; Lee, E.; Simister, E.; Thomas, R. The use of contrast variation in the specular reflection of neutrons from interfaces. Physica B: Condensed Matter 1991, $173,143-156$.

(76) Cubitt, R.; Fragneto, G. Scattering; 2002; pp 1198-1208.

(77) Saerbeck, T.; Cubitt, R.; Wildes, A.; Manzin, G.; Andersen, K. H.; Gutfreund, P. Recent upgrades of the neutron reflectometer D17 at ILL. Journal of Applied Crystallography 2018, 51, 249-256.

(78) Campbell, R. A.; Wacklin, H. P.; Sutton, I.; Cubitt, R.; Fragneto, G. FIGARO: The 
new horizontal neutron reflectometer at the ILL. European Physical Journal Plus 2011, $126,1-22$.

(79) Penfold, J. et al. Recent advances in the study of chemical surfaces and interfaces by specular neutron reflection. Journal of the Chemical Society - Faraday Transactions 1997, 93, 3899-3917.

(80) Nelson, A. R. Co-refinement of multiple-contrast neutron/X-ray reflectivity data using MOTOFIT. Journal of Applied Crystallography 2006, 39, 273-276.

(81) Nelson, A. R.; Prescott, S. W. Refnx: Neutron and X-ray reflectometry analysis in python. J. Appl.Cryst. 2019, 52, 193-200.

(82) Kučerka, N.; Nagle, J. F.; Sachs, J. N.; Feller, S. E.; Pencer, J.; Jackson, A.; Katsaras, J. Lipid Bilayer Structure Determined by the Simultaneous Analysis of Neutron and XRay Scattering Data. Biophysical Journal 2008, 95, 2356-2367.

(83) Junghans, A.; Köper, I. Structural analysis of tethered bilayer lipid membranes. Langmuir 2010, 26, 11035-11040.

(84) Kim, J.; Seidler, P.; Wan, L. S.; Fill, C. Formation, structure, and reactivity of aminoterminated organic films on silicon substrates. Journal of Colloid and Interface Science 2009, 329, 114-119.

(85) Biomolecular Scattering Length Density Calculator. http://psldc.isis.rl.ac.uk/ Psldc/.

(86) Appel, M.; Hizlan, D.; Vinothkumar, K. R.; Ziegler, C.; Kühlbrandt, W. Conformations of NhaA, the $\mathrm{Na} / \mathrm{H}$ Exchanger from Escherichia coli, in the pH-Activated and IonTranslocating States. Journal of Molecular Biology 2009, 386, 351-365.

(87) Schwörer, F.; Trapp, M.; Xu, X.; Soltwedel, O.; Dzubiella, J.; Steitz, R.; Dahint, R. Drastic Swelling of Lipid Oligobilayers by Polyelectrolytes: A Potential Molecular 
Model for the Internal Structure of Lubricating Films in Mammalian Joints. Langmuir 2018, 34, 1287-1299, PMID: 29251938.

(88) Schwörer, F.; Trapp, M.; Ballauff, M.; Dahint, R.; Steitz, R. Surface-Active Lipid Linings under Shear Load-A Combined in-Situ Neutron Reflectivity and ATR-FTIR Study. Langmuir 2015, 31, 11539-11548, PMID: 26388226.

(89) Killian, J. Hydrophobic mismatch between proteins and lipids in membranes. Biochimica et Biophysica Acta (BBA) - Reviews on Biomembranes 1998, 1376, 401-416.

(90) de Jesus, A. J.; Allen, T. W. The determinants of hydrophobic mismatch response for transmembrane helices. Biochimica et Biophysica Acta (BBA) - Biomembranes 2013, $1828,851-863$.

(91) Kim, T.; Lee, K. I.; Morris, P.; Pastor, R. W.; Andersen, O. S.; Im, W. Influence of hydrophobic mismatch on structures and dynamics of gramicidin a and lipid bilayers. Biophys J 2012, 102, 1551-60.

(92) Kandasamy, S. K.; Larson, R. G. Molecular dynamics simulations of model transmembrane peptides in lipid bilayers: a systematic investigation of hydrophobic mismatch. Biophys J 2006, 90, 2326-43. 


\section{Graphical TOC Entry}

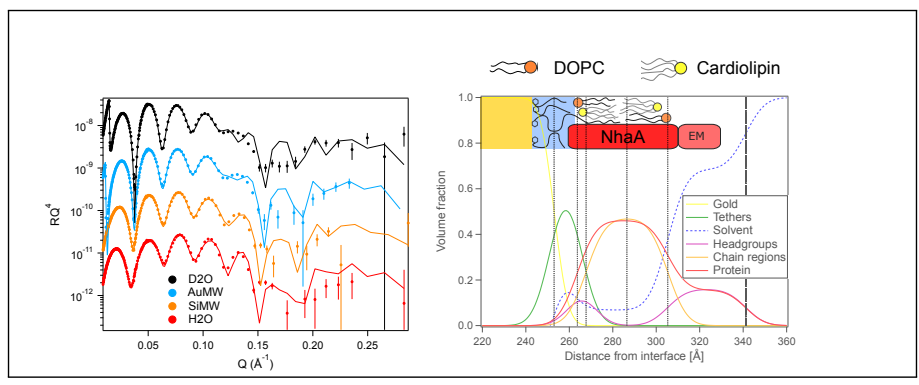

